\title{
Life history of an anticyclonic eddy
}

\author{
P. Sangrà, ${ }^{1}$ J. L. Pelegrí, ${ }^{2}$ A. Hernández-Guerra, ${ }^{1}$ Igor Arregui, ${ }^{3}$ J. M. Martín, ${ }^{1}$ \\ A. Marrero-Díaz, ${ }^{1}$ A. Martínez, ${ }^{1}$ A. W. Ratsimandresy, ${ }^{4}$ and A. Rodríguez-Santana ${ }^{1}$ \\ Received 11 June 2004; revised 5 November 2004; accepted 25 January 2005; published 23 March 2005.
}

[1] We use the trajectory of three buoys dragged below the surface mixed layer, together with sea surface temperature imagery, to examine the evolution of an anticyclonic warm-core eddy since its generation by the Canary Islands. Two buoys remain within the eddy during some 100 days, and the third one remains almost 200 days, while drifting southwestward up to $500 \mathrm{~km}$ with the mean Canary Current. The eddy merges with several younger anticyclonic and cyclonic eddies, in each occasion, suffering substantial changes. The eddy core, defined as a region with near-solid-body-type rotation and radial convergence, initially occupies the whole eddy. After interacting with another vortex the inner core markedly slows down, although it continues displaying radial convergence and relatively small radial oscillations, and an uncoupled outer ring is formed or enhanced, which revolves even more slowly and displays large radial fluctuations. The vortex extensive life is consistent with its inertially stable character and observations of radial convergence. A very simple model of vortex merging, where cylinders fuse conserving mass and angular momentum, gives fair results. The observations suggest that the eddy changes, as the result of its own slow evolution and sporadic mixing events, from a young stage, where the core retains its vorticity and occupies most of the eddy, through a mature stage, where the eddy has a reduced inner core and a slowly revolving outer ring, to a decay stage, where the vorticity maximum is substantially reduced.

Citation: Sangrà, P., J. L. Pelegrí, A. Hernández-Guerra, I. Arregui, J. M. Martín, A. Marrero-Díaz, A. Martínez, A. W. Ratsimandresy, and A. Rodríguez-Santana (2005), Life history of an anticyclonic eddy, J. Geophys. Res., 110, C03021, doi:10.1029/2004JC002526.

\section{Introduction}

[2] The generation of topographic eddies in a rotating fluid has received considerable attention during the last decades. A number of observational, numerical and laboratory studies have contributed to appreciate the structure and forcing mechanics of cyclonic and anticyclonic eddies near their generation area [Boyer and Kmetz, 1993; Chabert D'Hieres et al., 1989; Arístegui et al., 1994; Matsuura, 1995; Sangrà, 1995; Barton et al., 1998, 2000; Kennan and Flament, 2000; Flament et al., 2001]. However, there are still many open questions about the posterior temporal and spatial evolution of these eddies in the ocean. We do not know, for example, how long the topographically generated eddies last as coherent structures.

[3] The Canary Archipelago stretches zonally off northwest Africa, perturbing both the currents and surface winds

\footnotetext{
${ }^{1}$ Departamento de Física, Facultad de Ciencias del Mar, Universidad de Las Palmas de Gran Canaria, Las Palmas de Gran Canaria, Spain.

${ }^{2}$ Grup d'Oceanografía Física, Institut de Ciències del Mar, Centre Mediterrani d'Investigacions Marines i Ambientals, Consejo Superior de Investigaciones Científicas, Barcelona, Spain.

${ }^{3}$ Fundación AZTI, Pasaia (Gipuzkoa), Spain.

${ }^{4}$ Ente Público Puertos del Estado, Madrid, Spain.
}

Copyright 2005 by the American Geophysical Union. 0148-0227/05/2004JC002526\$09.00 in the region (Figures 1-3). The prevailing winds are the northeasterly trades, which intensify during summer, and the mean permanent current is the southwestward Canary Current. The lee side of the islands is a region sheltered from the prevailing northeasterly trade winds by their relief. As a consequence a warm surface wake, that stretches as much as $100 \mathrm{~km}$ Southwest from most of the islands, is usually noticeable [Hernández-Guerra et al., 1993; Barton et al., 2000]. Of major importance for our study is the central island of Gran Canaria, with near circular geometry and a clean exposure to the predominant southwestward Canary Current. It causes the frequent shedding of cyclonic and anticyclonic eddies, in a fashion that resembles a von Karman vortex street, and becomes the major source of mesoscalar variability south of the archipelago [Aristegui et al., 1994, 1997; Barton et al., 1998, 2000; Basterretxea et al., 2002]. Eddies have also been observed downstream from other islands but not as frequently as from Gran Canaria [Hernández-Guerra et al., 1993; Barton et al., 1998; Borges et al., 2004].

[4] In this study we examine the temporal and spatial evolution of an anticyclonic eddy found south of Gran Canaria through the analysis of the trajectory of three Argos buoys and with the help of sea surface temperature (SST) imagery. This zone is characterized by a mean gradient in the temperature of the surface waters, from the upwelling region into the interior ocean, and by the presence of intense 


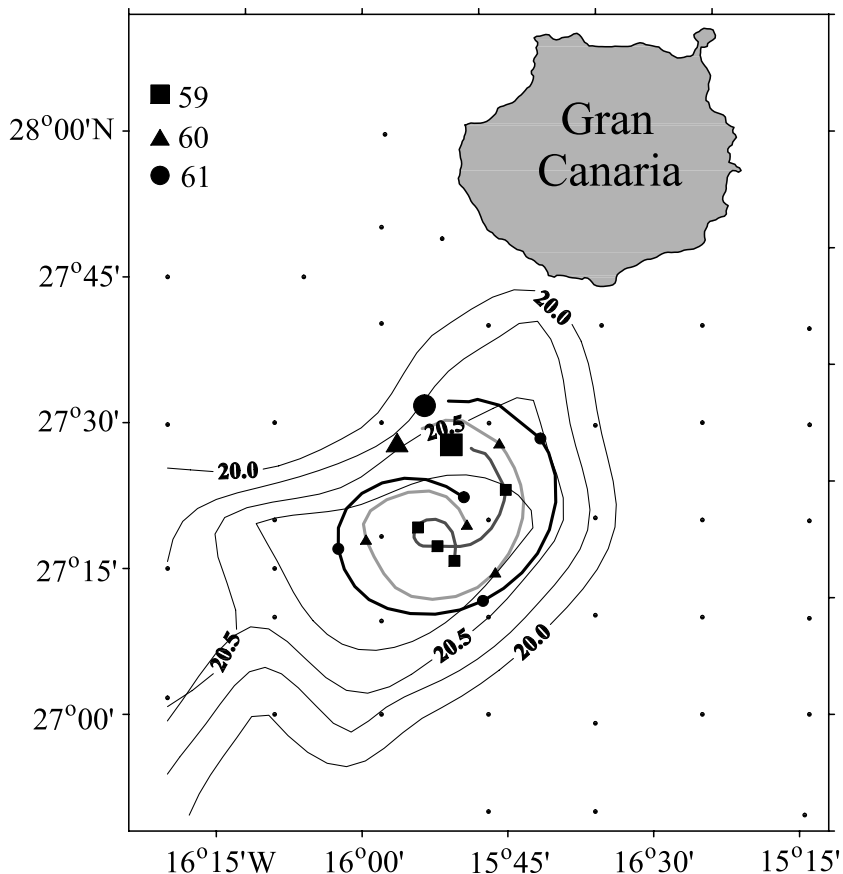

Figure 1. Buoy trajectories during the first 3 days after deployment superposed on the temperature field at $100 \mathrm{~m}$ depth. The buoys' deployment locations are indicated with large symbols, their position every day is indicated with the mid-size solid dots, and the XBT station positions are marked with small dots.

frontal regions associated with mesoscalar variability such as vortices and filaments generated in the coastal upwelling zone [van Camp et al., 1991]. Further, the anticyclonic (cyclonic) eddies shed by the islands produce warm (cold) surface water signals. The buoys' trajectories and their superposition onto the SST images will prove to be very helpful to examine the spatial and temporal coherence of the vortices shedding from the Canary Archipelago. In particular, SST images help identify those instances when the buoys' behavior arises from the interaction between the anticyclonic eddy and other mesoscalar structures.

[5] We also pursue a more quantitative description of the kinematics of water parcels through a careful analysis of the temporal evolution of the buoys' velocity fields, energy spectra, orbital radius and orbital periods. The buoys' trajectories, however, are a limited sample of the eddy so they can only give a partial view of its state. From this perspective we must clearly differentiate between the buoys' behavior, or regime, that depends strongly on the radial position of the buoy within the eddy, and the eddy stages, that reflect major changes in the eddy evolution. In our case the buoys' regime is initially related to the eddy stage because all three buoys were deployed within the eddy's core shortly after its generation, but the subsequent position and behavior of these buoys changes drastically as the eddy interacts with other mesoscalar structures.

[6] During the last 2 decades substantial understanding has been attained on issues such as vortex shedding periodicity, vortex persistence, and the evolution of the eddy as it interacts with other mesoscalar structures [e.g., Cushman-Roisin et al., 1985; Hogg and Stommel,
1985; Flierl, 1987, 1988; Kloosterziel and van Heijst, 1991; Hopfinger and van Heijst, 1993; Matsuura, 1995; Carnevale et al., 1997]. Most analytical, numerical, or laboratory studies, however, consider rather idealized conditions which may lead to dissimilar answers on apparently basic issues, such as whether cyclonic an anticyclonic geophysical vortices have similar behavior and what is the role of barotropic and baroclinic instabilities for eddies of different size and relative vorticity. The combined analysis of buoy and SST data should prove helpful to look at some of these topics. In next section we introduce the available data, and in section 3 we examine the buoys' instantaneous and mean trajectories, and explore their relation with SST imagery to identify when the eddy interacts with other features. In section 4 we examine in detail the orbital motions, looking at the temporal evolution of their kinetic energy, orbital period and radius. We leave to section 5 the interpretation of the information provided by the trajectories, examining questions such as the stability of the vortex, shedding frequency, vortex merging, and long-term evolution. We sum up the main conclusions in section 6 .

\section{Buoy and Sea Surface Temperature (SST) Data}

[7] In June 1998 an interdisciplinary survey was carried out on the lee side of Gran Canaria island with R/V Garcia del Cid and A/V Las Palmas. The survey was planned to study the physical and biological properties of the warm wake and an anticyclonic eddy that had recently detached from the island. The presence of these structures was initially confirmed with SST satellite imagery, and hydrographic sections were carried out from the R/V García del Cid to locate the eddy center. The anticyclonic eddy was initially about $50 \mathrm{~km}$ in diameter, comparable to the island's size, being characterized by a $60 \mathrm{~m}$ downwelling of the central isopycnals and by a core of relatively warm surface water (see details in the work of Basterretxea et al. [2002]). The downwelling of the isopycnals is maintained throughout the upper thermocline layers, which consist on North Atlantic Central Waters that flow south as the Canary Current [Arístegui et al., 1994; Pérez et al., 2001; Basterretxea et al., 2002]. On 29 June three buoys were deployed within the eddy, at different radial distances from its center, from the R/V Garcia del Cid. The buoys' drogues were set at $100 \mathrm{~m}$ depth, below the surface mixed layer, in order to properly track the upper thermocline flow. Two of the buoys (buoys 59 and 61) were deployed with a holey sock drogue and the other one (buoy 60) with a semispherical drogue. Gasser et al. [2001] performed a field testing on the behavior of the two different drogue types, concluding that the semispherical drag area ratio was as efficient as the holey sock one.

[8] All three buoys followed the eddy over 100 days: buoy 59 remained 104 days (30 June-12 October), buoy 60 stayed 102 days (30 June-10 October), and buoy 61 tracked it during 199 days (30 June 1998-14 January 1999). The buoys continued transmitting beyond these times, but their tracks did not longer display any sort of revolution so we concluded that they had left the eddy. We received an average number of about ten positions per day for each buoy. The positions are interpolated every 3 hours using a polynomial fit and the raw data are smoothed with a 
(a)

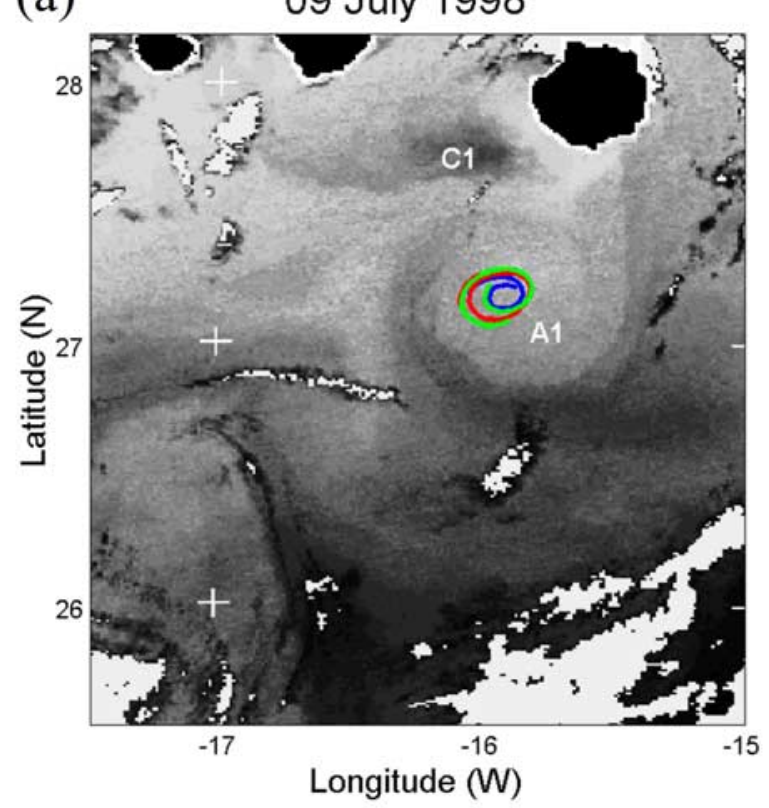

(c)

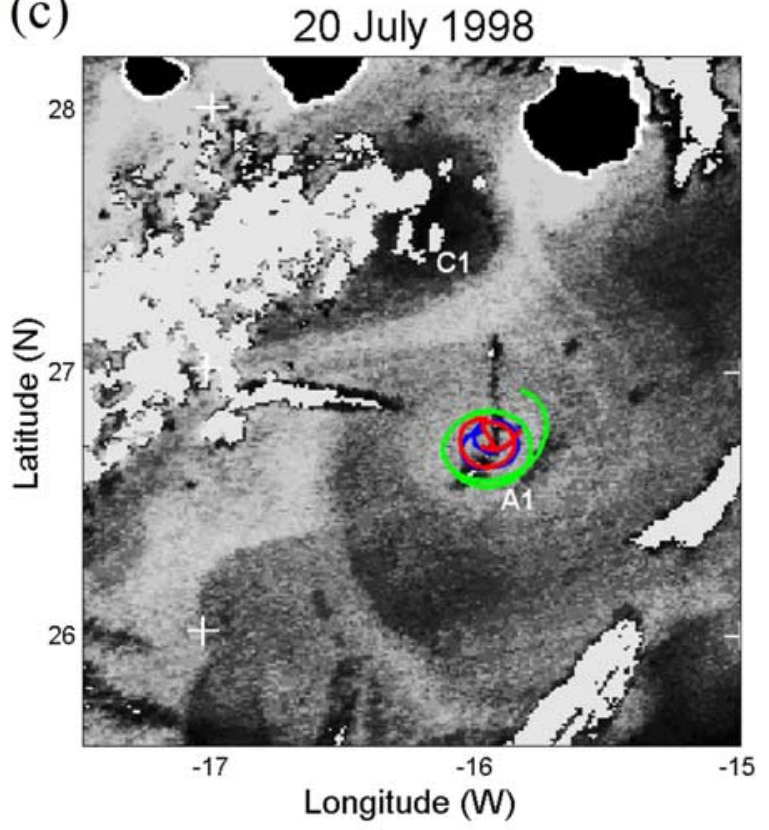

(b) 11 July 1998

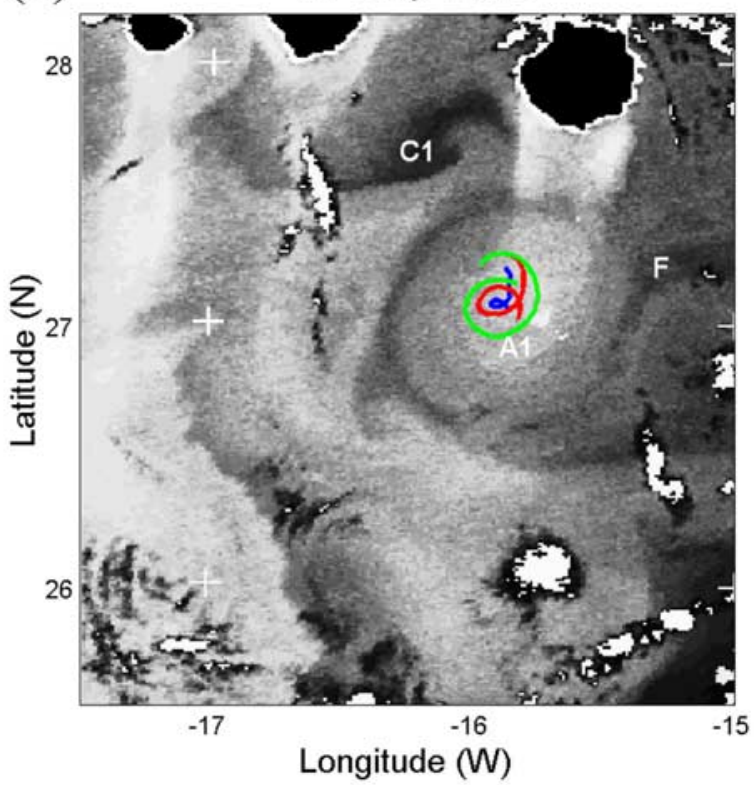

(d)

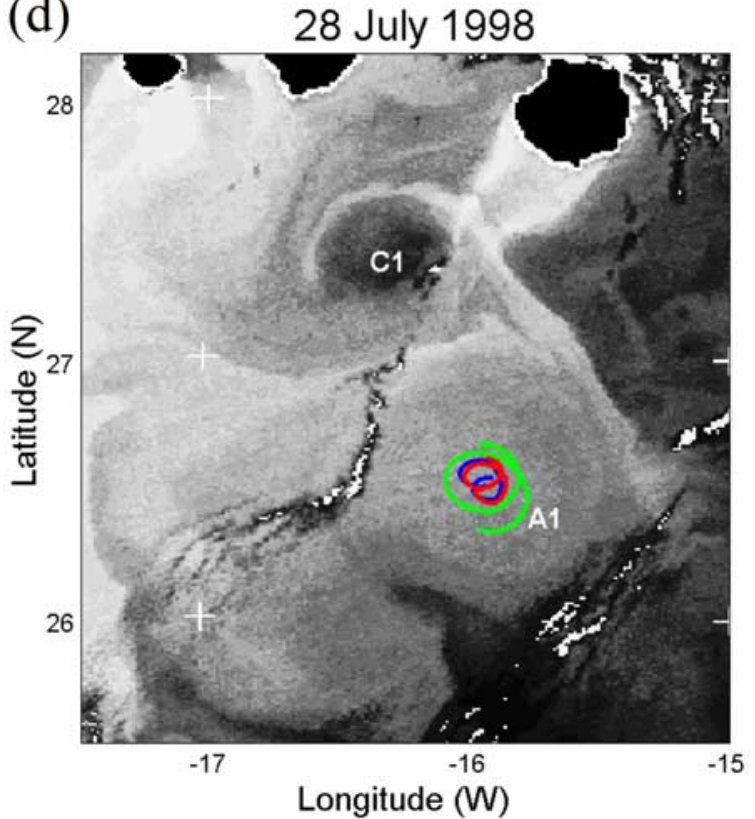

Figure 2. Trajectories for all three buoys superposed onto sea surface temperature (SST) images: (a) days 8-11 superposed to day 9 (9 July); (b) days 11-14 superposed to day 11 (11 July); (c) days 18 22 superposed to day 20 (20 July); (d) days 28-32 superposed to day 28 (28 July); (e) days 45-50 superposed to day 47 (16 August); (f) days 53-58 superposed to day 55 (24 August); (g) days 69-75 superposed to day 72 (10 September); (h) days 88-94 superposed to day 91 (29 September); (i) days 114-122 superposed to day 118 (26 October); and (j) days 138-146 superposed to day 142 (19 November). Insets zoom at the buoys' trajectories; the color code is blue for buoy 59, red for buoy 60 , and green for buoy 61 . 

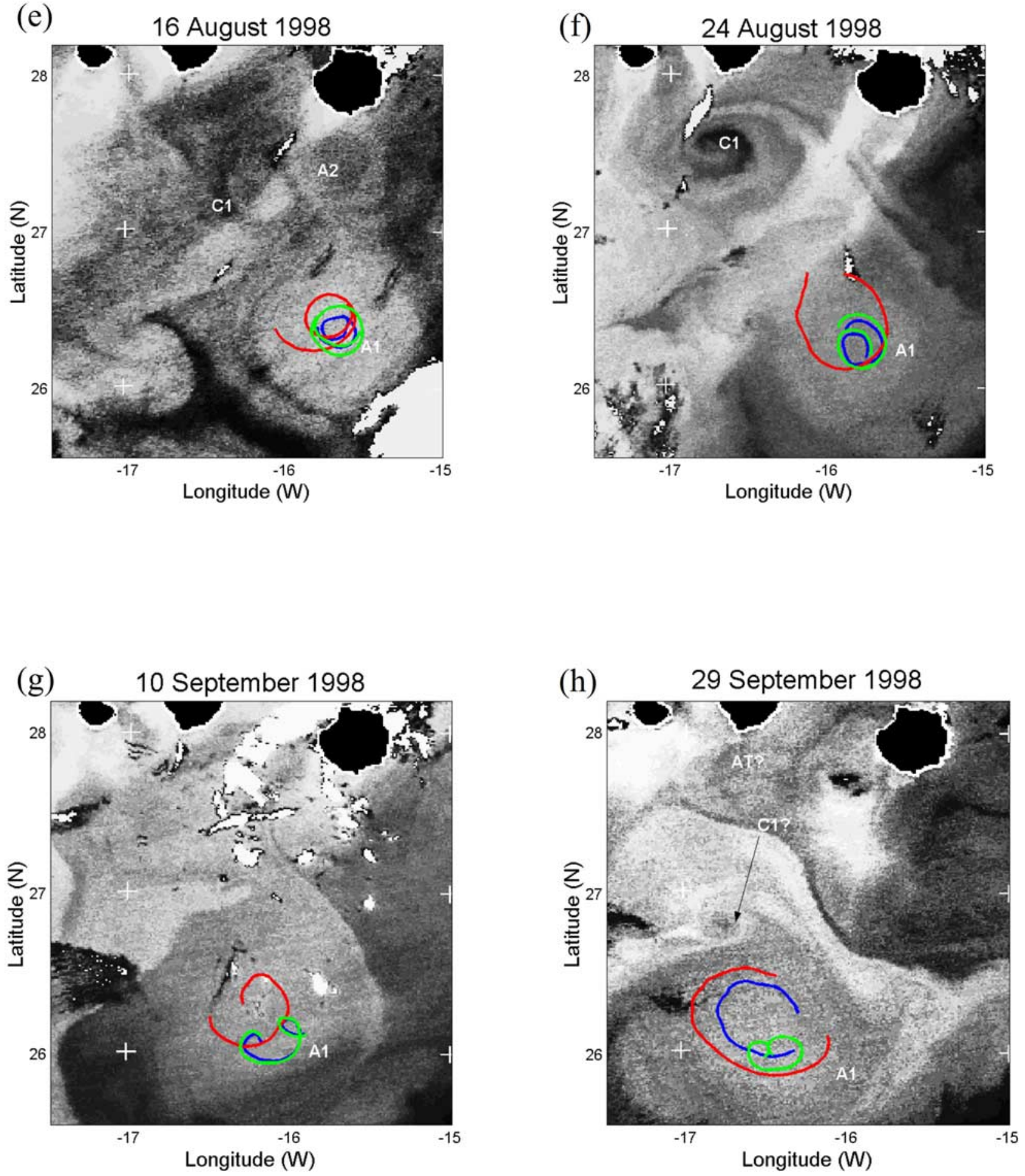

Figure 2. (continued)

12 hour low-pass Fourier filter [e.g., Press et al., 1986, p. 495]. This filter is very efficient to remove high frequencies (periods below 12 hours) but it does not remove any potential contributions from the tidal or inertial oscillations (26.5 hour period in the eddy's generation area). However, we will see below that the tidal and inertial contributions are very minor because of the $100 \mathrm{~m}$ depth droguing (that removes most inertial contributions within the surface mixed layer) and because of the small open-ocean tidal signal [Dick and Siedler, 1985; Siedler and Paul, 1991]. Since the signal we are interested has periodicities as low as 2 days we have run some tests using a 36 hours low-pass Fourier filter and found that it actually gets rid not only of the energy at 1 day periods but also substantially modifies the shape of the energy spectra at periods close to 2 days. For this reason we have decided to only use the 3 hour 


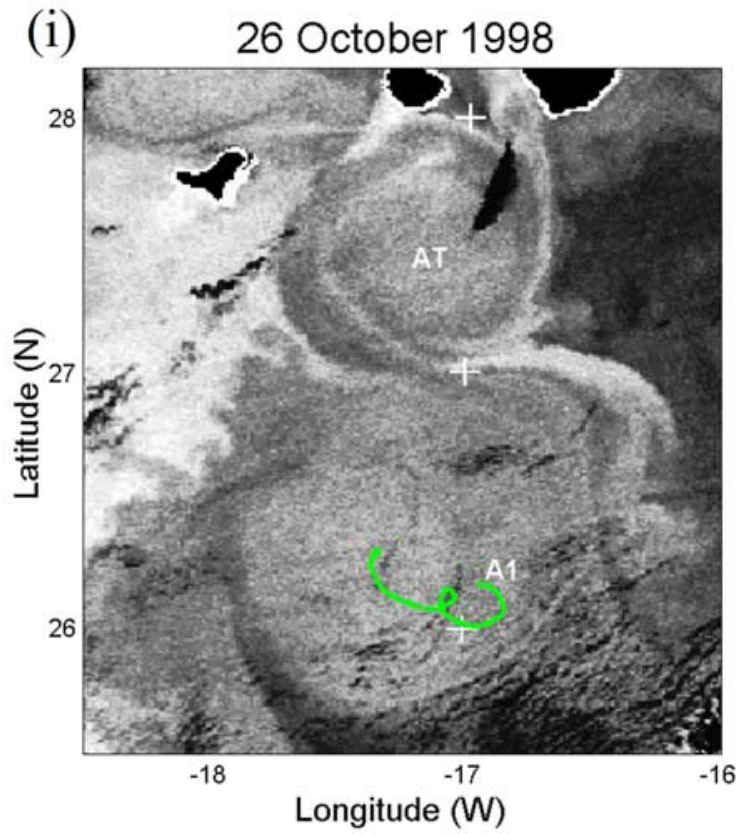

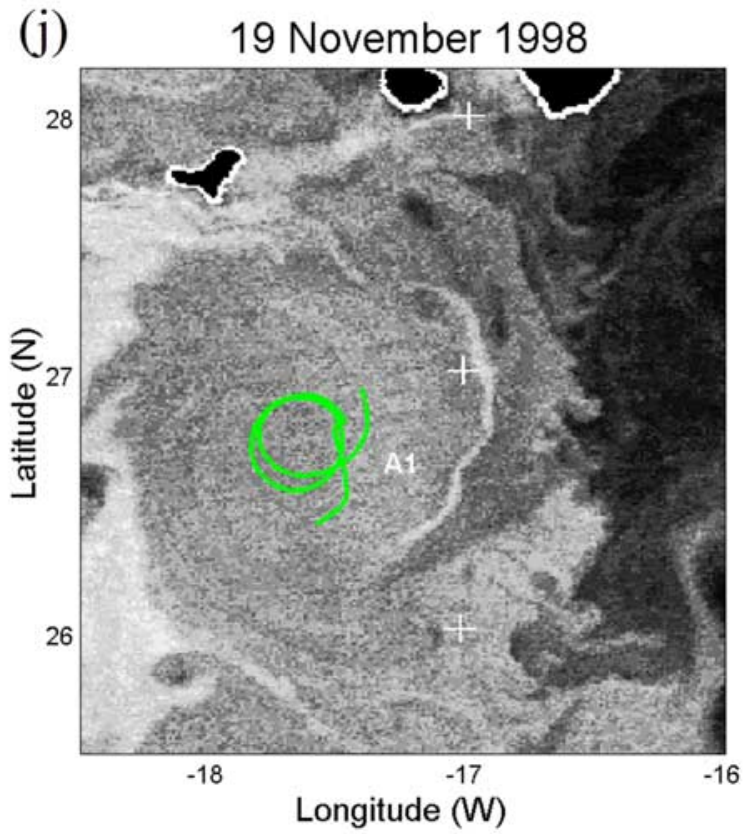

Figure 2. (continued)

interpolation and 12 hour low-pass Fourier filter to obtain the buoys' trajectories and instantaneous velocity time series.

[9] Figure 1 indicates the position where the buoys were launched and illustrates the initial trajectories during 3 days superposed on the temperature field at $100 \mathrm{~m}$ depth. Midsize symbols are shown every 24 hours to indicate the buoys' positions at the beginning of every day. The trajectory between the deployment and the first symbol is provided by the first several raw position fixes and thereafter by the interpolated positions. Buoys 59, 60 and 61 initially follow internal, intermediate and external clockwise open orbits, respectively. The temperature data were obtained on an XBT grid conducted with the A/V Las Palmas between 29 June and 1 July. For the sake of clarity only those isotherms higher than $20^{\circ} \mathrm{C}$ are shown. The temperature field displays a warm-core structure that corresponds to the anticyclonic eddy south of Gran Canaria. The eddy has an elongated shape but this is the result of the southwestward current, as shown by the simultaneous drift of the buoys, and the sampling strategy, starting at the northeastern corner and following west by columns.

[10] We have also examined all SST images available from NOAA satellites for this region during the time period the buoys traced the eddy. SST images were acquired through a local satellite receiving station located in Gran Canaria, and processed using the methodology presented by Eugenio et al. [2001]. Relatively warm surface temperature is expressed as light grey tones while dark tones correspond to relatively cold water. Because of cloud coverage the SST sequence is not complete but the available images allow us to appreciate the major features during the vortex evolution. The images illustrate intense temperature contrasts, which reflect the strong mesoscalar activity resulting from both coastal upwelling and topographic forcing of the Canary
Current. The intensity of some warm features, such as the lee wakes detaching from the islands, may depend on the time of the day when the image was taken and the state of the sea. If the image was taken on the afternoon of a calm area then SST increases because of the formation and preservation of a diurnal warm layer.

\section{Trajectories}

[11] The association between the near-surface temperature field and the buoy motion is clear in Figure 1, where the initial trajectories are related to the warm core. The buoys move in phase resembling solid-body-type rotation, with a 2.5 day revolution period, but their motion does not result in a closed orbit because the eddy drifts with the southwestard mean flow. We may use the SST imagery, in conjunction with the buoy trajectories, to obtain a good visualization of the southward propagating eddy. In order to ease identify the time elapsed we consider 0 hours of 30 June 1998, the deployment date, as day 0 . Notice, however, that the eddy was possibly generated about 2 weeks earlier [Basterretxea et al., 2002].

[12] In Figure 2 we present a total of ten images that go from day 9 to day 142 after deployment. On these images we have superposed the buoys' trajectories for several days, approximately centered at the time of the satellite image, and have identified the most relevant mesoscalar structures. The images are presented by pairs, with each image of a pair typically a few days apart. The temporal closeness between images in each pair helps to visualize the short-term evolution of the temperature field, while the longer time between adjacent pairs ( $1-4$ weeks) provides a view of the long-term evolution.

[13] Figure 2a shows the trajectories between days 8 and 11 superposed on the day 9 (9 July) image. All three buoys follow near circular trajectories over the surface warm water 
(a)

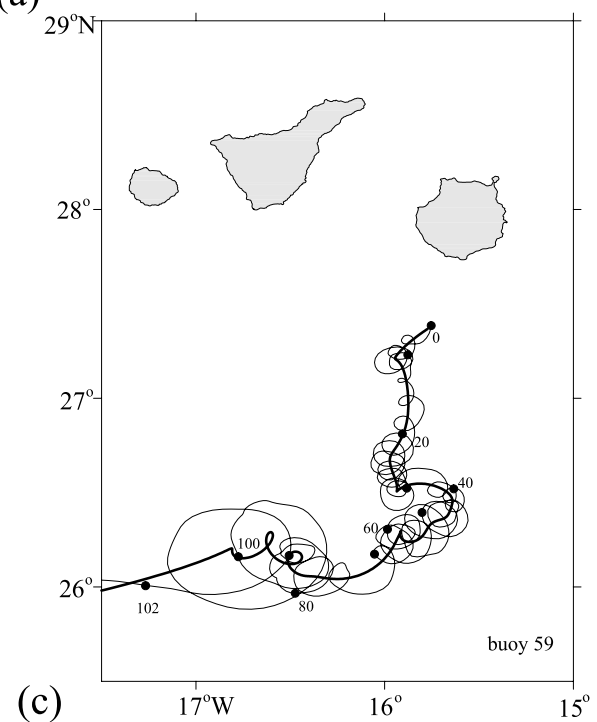

(b)

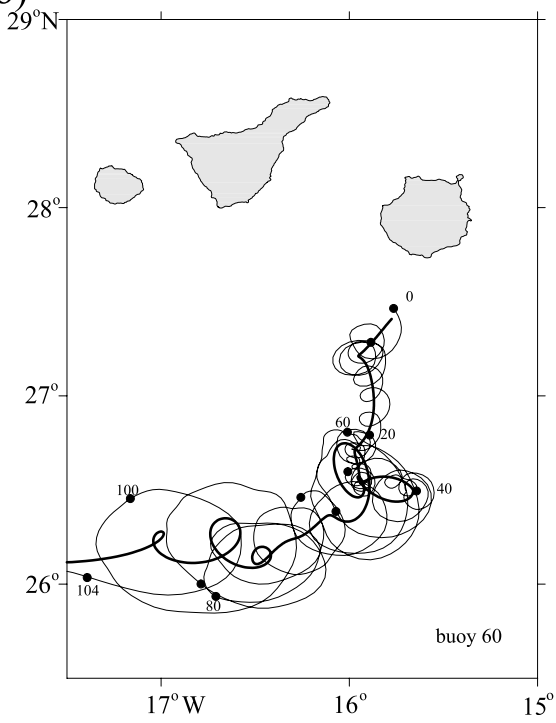

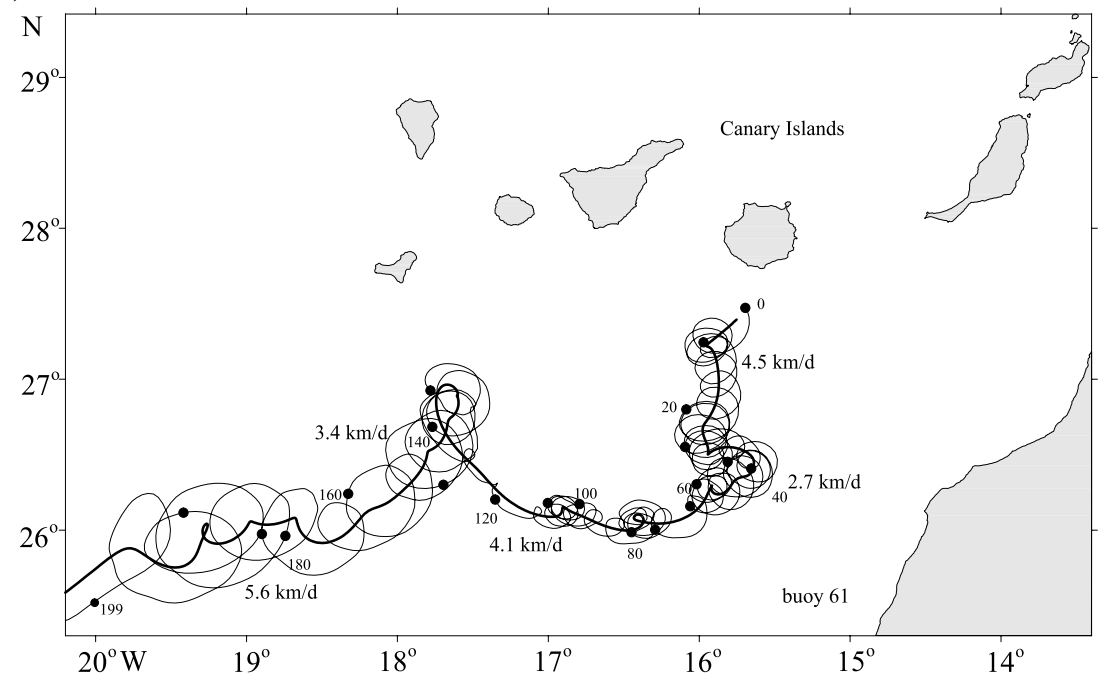

Figure 3. Temporal evolution of the position of the eddy center as traced by each buoy (bold line) superposed onto the full buoy trajectory within the eddy (thin line): (a) buoy 59 (days $0-104$ correspond to 30 June-12 October); (b) buoy 60 (days $0-102$ correspond to 30 June-10 October); (c) buoy 61 (days 0-199 correspond to 30 June 1998-14 January 1999). In Figure 3c we also indicate the mean speed of the vortex center. The numbers indicate the days (multiples of 20) elapsed after the buoys deployment, and dots are drawn every 10 days.

core A1, suggesting very small background velocities. Figure $2 \mathrm{~b}$ illustrates the trajectories from day 11 to 14 superposed on the day 11 (11 July) image. The trajectories are further stretched, as compared with Figure $2 \mathrm{a}$, which indicates a significant southwestward mean flow. In Figures $2 \mathrm{a}$ and $2 \mathrm{~b}$ we appreciate several other mesoscalar structures, in particular relatively cold waters corresponding to a cyclonic vortex $\mathrm{C} 1$ and an upwelling filament $\mathrm{F}$. The cyclonic vortex is located adjacent to the southwest coast of Gran Canaria, suggesting a very recent generation. The filament stretches offshore from the African coast and draws the anticyclonic eddy contour. A third type of structure visible in these and the following figures are the warm lee wakes that stretch southwest from several islands, specially Gran Canaria, in the direction of the prevailing wind [Hernández-Guerra et al., 1993; Barton et al., 2000].
[14] Figure 2c shows the trajectories between days 18 and 22 superposed on the day 20 (20 July) image, and Figure $2 \mathrm{~d}$ illustrates the tracks between days 28 and 32 on the day 28 (28 July) image. During this whole period the buoys slowly drift south, in solid body type rotation, tracking the anticyclonic eddy. The entrainment of warm water from the island's wake enhances A1, a high-temperature signal. Cyclone $\mathrm{C} 1$ has been advected southwest and the vortex pair $\mathrm{A} 1+\mathrm{C} 1$ resembles a portion of a von Karman vortex street generated downstream of an obstacle. The two images clearly show that in just 1 week the two opposite sign vortices have considerably approached each other.

[15] Figure 2e shows the paths between days 45 and 50 superposed on the day 47 (16 August) image, and Figure $2 \mathrm{f}$ draws the tracks between days 53 and 58 superposed on the day 55 (24 August) image. During this full time interval the 
(a)

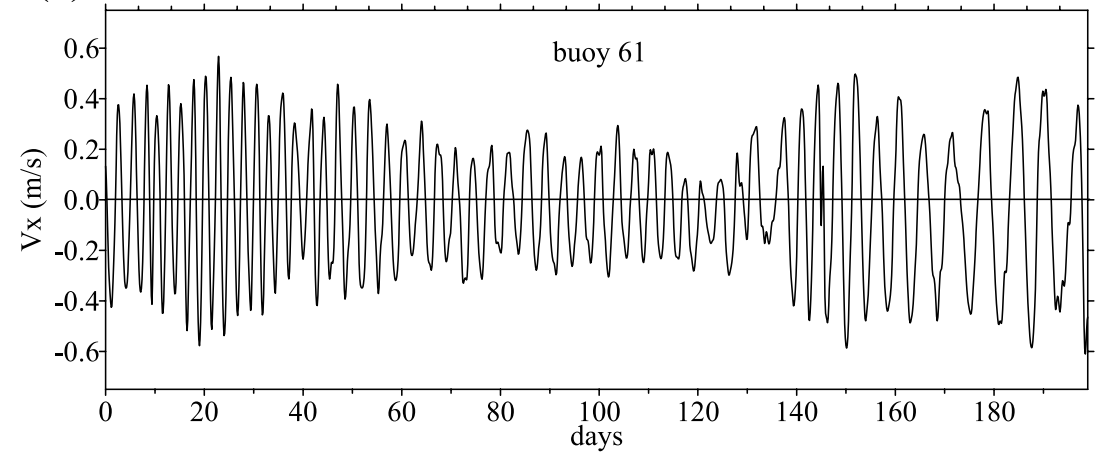

(b)

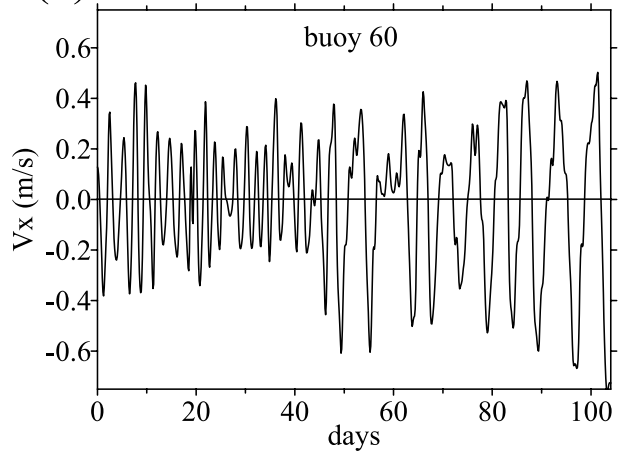

(c)

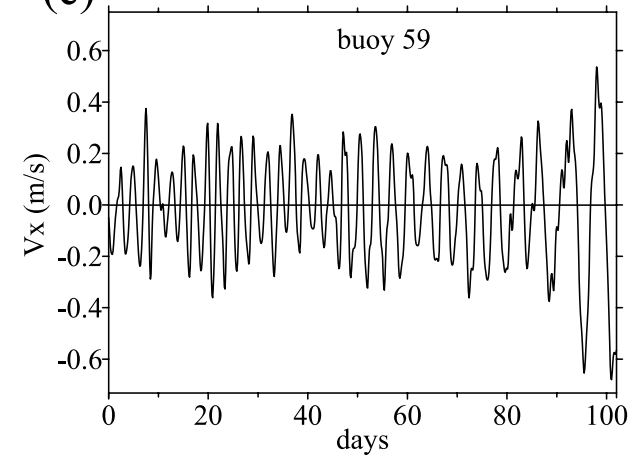

Figure 4. Zonal velocities (eastward positive) for all three buoys during the time intervals they remained within the eddy. Day 0 corresponds to 30 June 1998. (a) Buoy 61. (b) Buoy 60. (c) Buoy 59.

eddy remains almost stationary, with the buoys drawing near closed orbits over a broad SST signal. Figure 2e shows that buoy 60 , originally the intermediate buoy, approximately triples its radius and becomes the exterior buoy, while buoy 59, the interior one, doubles its radius and joins buoy 61. Figure $2 \mathrm{f}$ clearly illustrates buoy 60 rotating much slower than the other two buoys. Figure $2 \mathrm{e}$ exhibits a second warm core structure A2 that has just formed south of Gran Canaria. The cyclonic eddy $\mathrm{C} 1$ does not show up very clearly but a Sea-viewing Wide Field-of-view Sensor (SeaWiFS) color image for 19 August (not shown) confirms the presence of a high-pigment patch precisely at this location. Figure 2e also shows that vortices $\mathrm{C} 1$ and $\mathrm{A} 1$ have swiftly drifted apart, possibly following a repulsion between opposite sign vortices (Figure 2d). Figure $2 \mathrm{f}$ suggests that anticyclonic eddy $\mathrm{A} 2$ is just merging with $\mathrm{A} 1$, and that it has driven vortex $\mathrm{C} 1$ further west.

[16] Figure $2 \mathrm{~g}$ illustrates the paths of all buoys between days 69 and 75 superposed on the day 72 (10 September) image and Figure $2 \mathrm{~h}$ exhibits their trajectories between days 88 and 94 superimposed on the day 91 (29 September) image. The buoys' trajectories show that the vortex, after interacting with $\mathrm{C} 1$ and merging with $\mathrm{A} 2$, has resumed a net westward displacement. In Figure $2 \mathrm{~h}$ buoy 59 , initially the interior buoy, has moved radially out and is now describing, together with buoy 60 , a wide and relatively slow path, while buoy 61 revolves swiftly in the interior position. At this time (see next section) the rotation period of the external buoys has become approximately twice (about 5 days) the period of rotation of the internal buoy. In Figure $2 \mathrm{~g}$ we no longer appreciate $\mathrm{C} 1$, possibly because of surface capping through atmospheric heating, but Figure $2 \mathrm{~h}$ displays a rather small cyclonic (cold-core) feature that appears to be rolling just on top of A1, which may be an indication of some interaction between $\mathrm{A} 1$ and $\mathrm{C} 1$. In Figure $2 \mathrm{~h}$ a second warm-core vortex AT appears to be forming just south of Tenerife island (pear-shaped large island immediately west of Gran Canaria).

[17] Buoys 59 and 60 describe an outer trajectory until approximately day 102, when they leave the eddy. At this time, however, buoy 61 still remains tracing an anticyclonic loop that proves the eddy remains alive. Figure $2 \mathrm{i}$ illustrates its trajectory from day 114 to day 122 (22-30 October) superposed on the day 118 (26 October) image, and Figure $2 \mathrm{j}$ presents the trajectory between days 138 and 146 (15-23 November) superposed on the day 142 (19 November) image. In Figure $2 \mathrm{i}$ the anticyclonic eddy AT is quite evident immediately north of A1. The two nearby vortices begin to interact, with A1 moving northwest (as inferred by the mean buoy trajectory and the 4 November image, not shown) and AT southeast. In Figure $2 \mathrm{j}$ merging is completed, AT is visible no longer and A1 (as defined by the warm SST core) has increased its size and moved northwest. At this time the buoy has already resumed its southwest trajectory.

[18] Figure 3 illustrates the trajectories for all three buoys during the whole period they remained within the eddy. Buoys 59 and 60 initially maintain solid-body-type rotation, with a period of about 2.5 days and approximate constant orbital radius. Buoys 60 (about day 40) and 59 (about 
day 80) move away from the eddy center (tracked by buoy 61 ) and their orbital revolution rate decreases (Figures 3a and $3 \mathrm{~b}$ ). Buoy 61 maintains near-constant rotation rate until about day 135, afterward evolving in a similar pattern as the other two buoys (Figure 3c). The sequential escape of the buoys from the eddy core appears related to the interaction of the eddy with other vortices.

[19] Figure 3 also shows the paths followed by the vortex center, or mean buoy drift, as tracked by the three buoys. These center trajectories are obtained by applying the Fourier low-pass filter (same as described in section 2) over the 3 hour data positions, now with a 6 day low-pass Fourier filter. This cut-off period has been chosen as the smallest one that eliminates the orbital motions without unnecessarily smoothing the trajectories (see section 4). A comparison of the buoys' mean drifts shows good resemblance during the first 100 days, although there is some mismatch after about day 40 when the eddy interacts with other vortices. From days 0 to 60 the eddy center moves south with a mean speed ranging between $4.5 \mathrm{~km} \mathrm{~d}^{-1}$ (days 0-20) and $2.7 \mathrm{~km} \mathrm{~d}^{-1}$ (days 40-60). Between days 60 and 130 the eddy center moves west with a mean speed of about $4.1 \mathrm{~km} \mathrm{~d}^{-1}$. After day 130 the eddy center rapidly moves southwest, its mean speed increasing from 3.4 to $5.6 \mathrm{~km} \mathrm{~d}^{-1}$ during this time interval. These speeds (in the range between 3.1 and $6.5 \mathrm{~cm} \mathrm{~s}^{-1}$ ) are typical for the upper layers of the southwestward flowing Canary Current [Hernández-Guerra et al., 2001, 2002]. It suggests that this current is the main responsible for the drift of the eddy center, although some of changes in magnitude and direction at relatively short timescales (order 10 days) may be related to the interaction between vortices. Another factor that may lead to a net westward eddy displacement is the variation of the Coriolis parameter with latitude [Nof, 1981; Pingree and Sinha, 2001].

\section{Orbital Motions}

[20] A view of the temporal evolution of the eddy rotation rate may be obtained from either Cartesian component of the buoys' horizontal velocities. Figure 4 shows the zonal component of velocity (eastward positive) for all three buoys as obtained from the 12 hour smoothed time series. An analogous plot obtained using the meridional velocity component looks almost identical (but phase shifted) and is not shown. The evolution of the zonal velocity contains a great deal of information that will be discussed along this paper, with the important attribute that the data undergoes very little processing. At this point we stress two main features that repeat themselves throughout the different data analyses. The first one is that each buoy experiences at least two very different rotation regimes, the transition between them being characterized by major changes in the velocity amplitude and period. We will see below that these transitions stand out in all variables, besides other smaller transitions in the data record. The second feature (from buoys 60 and 61) is that the oscillation in the zonal speed decreases in amplitude after the first 2 or 3 weeks, but previous to the above mentioned major transition. Despite this speed reduction the rotation period remains approximately constant, which indicates that the buoys approach the eddy center. (a)
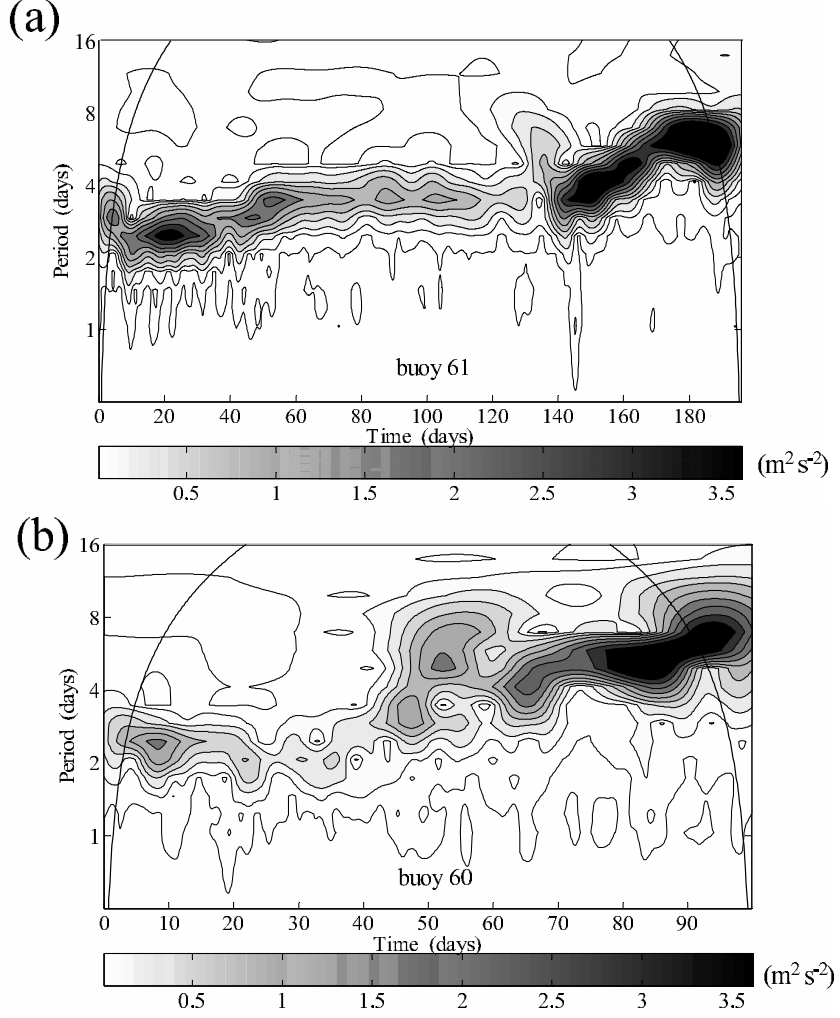

(c)

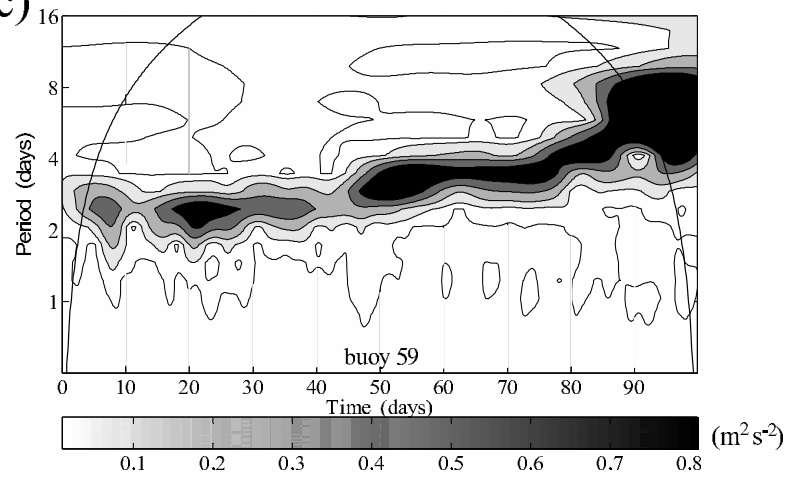

Figure 5. Kinetic energy spectra as a function of time as obtained from the wavelet analysis of the zonal velocities (note the geometric scale in the ordinate axis). The curved lines at both extremes of the time series indicate those regions where boundary effects decrease the confidence of the method. (a) Buoy 61. (b) Buoy 60. (c) Buoy 59.

[21] The zonal velocity time series may be used to determine the kinetic energy spectrum for each buoy (not shown). All buoys exhibit a low period peak located at approximately 2.5 days, which is attributed to the initial high rotation rate. There are several low-frequency energy peaks, at variable periods up to 8 days, possibly related to the dominant frequencies after the regime transition. These Fourier analyses, however, use the whole time series for each buoy, so they are unable to discriminate if the time series is split into two or more separate portions with distinct rotation rates. In order to remove this limitation we have done a wavelet analysis of the zonal velocities [Morlet et al., 1982; Grossmann and Morlet, 1984; 
(a)

(b)

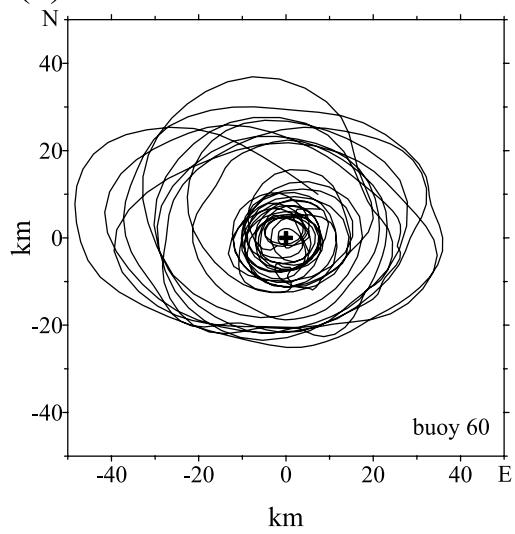

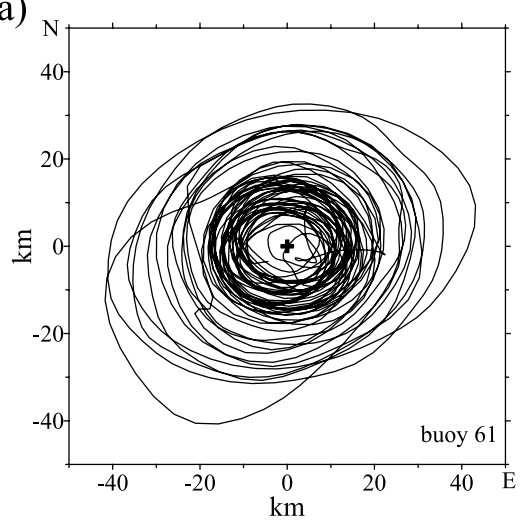

(c)

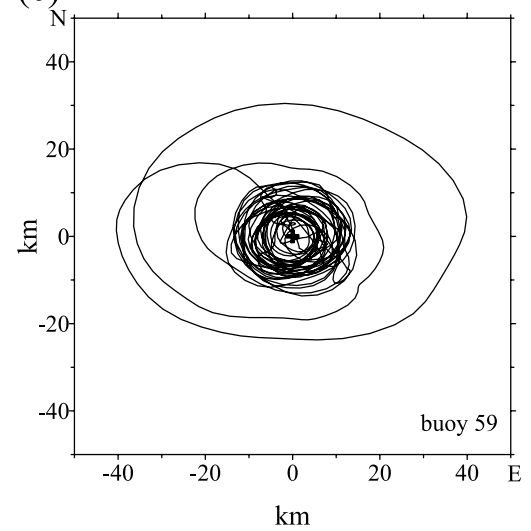

Figure 6. Buoys' orbits corresponding to the complete buoys' time series. (a) Buoy 61. (b) Buoy 60 . (c) Buoy 59 .

Grossmann et al., 1985] (see Percival and Walden [2000] for an updated review). The wavelet power spectrum is defined as the squared absolute value of the wavelet transform and gives a measure of the temporal evolution of the time series variance $\left(\sigma^{2}, \mathrm{~m}^{2} \mathrm{~s}^{-2}\right)$ for all periods (Figure 5). The contours correspond to kinetic energy density, in exactly the same manner as the energy spectra, and provide information on the energy level at each frequency. The sharpness of the peak energy is indicative of the stability of the rotation rate, while a peak that remains sharp but decreases in intensity reflects a smaller rotation radius.

[22] In Figure 5a, corresponding to buoy 61, we may separate the time domain of the energy spectra into several regions. During the first few days the dominant period is about 3.0 days but by day 10 this value has decreased and stabilized at 2.5 days. This period remains almost constant until approximately day 35 , during this time interval the peak energy being rather sharp and intense. After day 35 the buoy takes a couple of weeks for its period to change from about 2.5 to 3.6 days. The satellite images suggest that during this time interval the vortex repels $\mathrm{C} 1$ and merges with A2 (Figures 2d-2f). From day 50 till day 130 the dominant period remains stably centered at 3.6 days, although an oscillatory pattern starts at about day 85 (when A1 merges with C1, Figure $2 \mathrm{~h}$ ). During this time interval the peak energy decreases with time, as a result of the buoy approaching the eddy center, and becomes less sharp. At approximately day 130 the buoy's rotation rate is again modified, the satellite images suggesting this is the result of merging with anticyclonic eddy AT (Figures $2 \mathrm{i}$ and $2 \mathrm{j}$ ). The buoy temporally recovers the dominant rotation rate of 3.6 days and the peak energy increases, but the rotation rate progressively decreases until about day 175 when it reaches a period of 6.4 days.

[23] In Figure 5b, corresponding to buoy 60, the time domain of the energy spectra also shows several distinct regimes. Since its deployment until day 35 the dominant rotation period decreases slightly (from 2.4 to 2.0 days) and the level of maximum energy broadens. Beyond day 35 the rotation period increases briefly to almost 6 days (day 50) and stabilizes at about 4 days (after day 65), in a rather complex response to the repulsion between $\mathrm{A} 1$ and $\mathrm{C} 1$ and the merging of A1 and A2. From day 65 to day 85 the peak energy increases and the dominant period slowly changes from 4.0 to 5.5 days. At approximately days $85-90$ the rotation period increases markedly with time (during the interaction of A1 with $\mathrm{C} 1$, Figure $2 \mathrm{~h}$ ).

[24] Figure 5c illustrates the results of the wavelet analysis for buoy 59. Alike buoys 60 and 61 the rotation period remains quite constant till day 35, at about 2.5 days. During this time interval the peak energy is rather sharp although its intensity decreases with time. The transition between days 35 and 50 (related to the successive interaction between $\mathrm{A} 1$ with $\mathrm{C} 1$ and $\mathrm{A} 2$, Figures $2 \mathrm{~d}-2 \mathrm{f}$ ) is characterized by an increase in dominant period (from 2.5 to 3.4 days) and the recovery of the peak energy level. Between days 50 and 80 the dominant period is almost constant at 
(a)

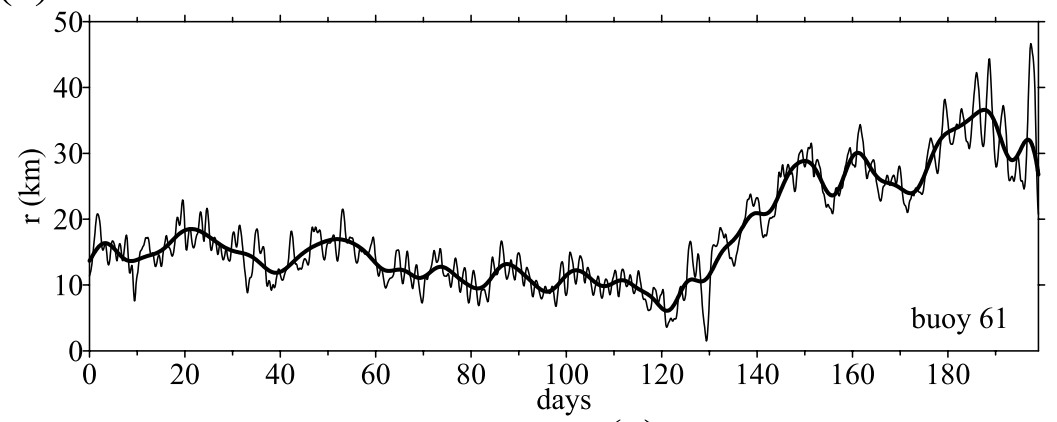

(b)

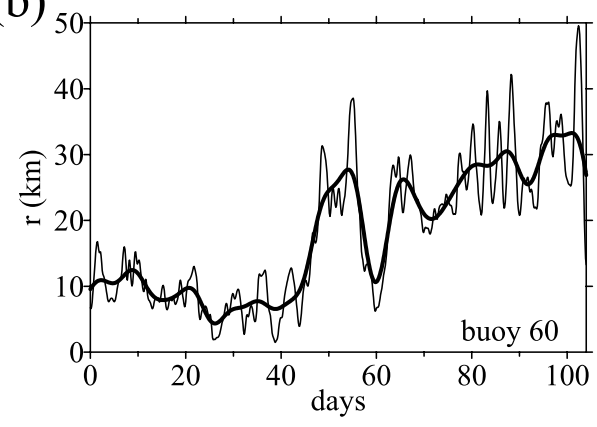

(c)

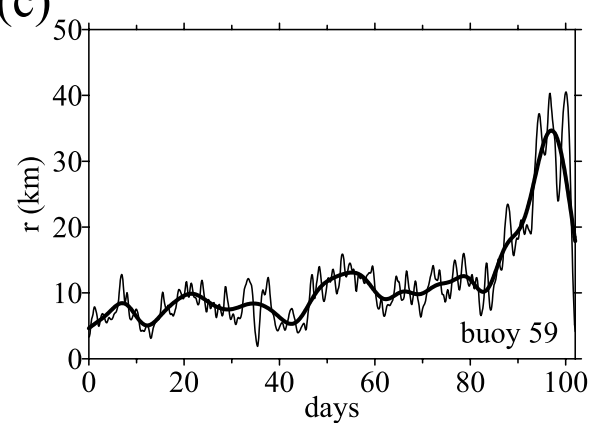

Figure 7. Instantaneous (thin line) and mean (bold line) orbital radius for (a) buoy 61, (b) buoy 60, and (c) buoy 59 .

about 3.4 days and the maximum energy level remains high. Following the interaction of A1 with $\mathrm{C} 1$ (day 80) the rotation period increases to $\sim 6$ days, the energy peak broadens and its intensity decreases (Figure $2 \mathrm{~h}$ ).
[25] A buoy's orbit may be calculated extracting the (6 day) mean buoy drift to the buoy trajectory. Figure 6 presents the orbits for all three buoys during the whole length of each time series. The orbit pattern is similar for all

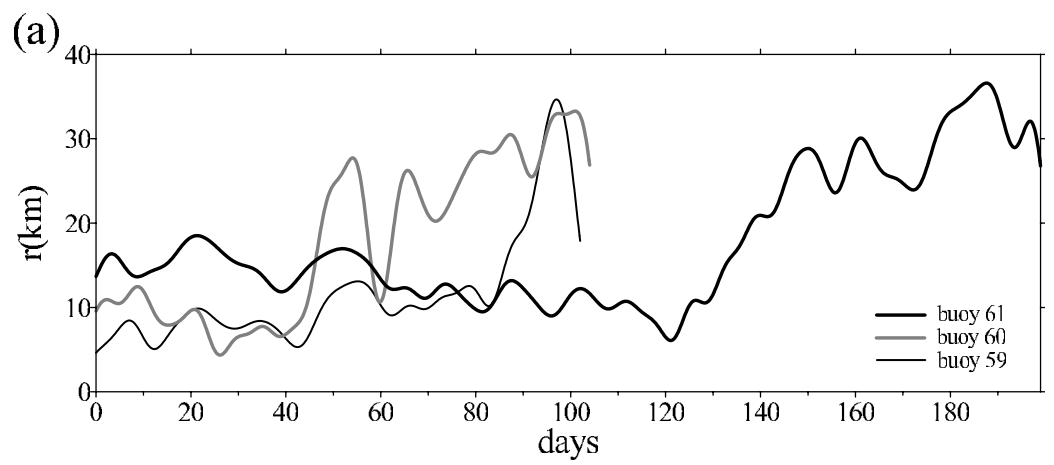

(b)

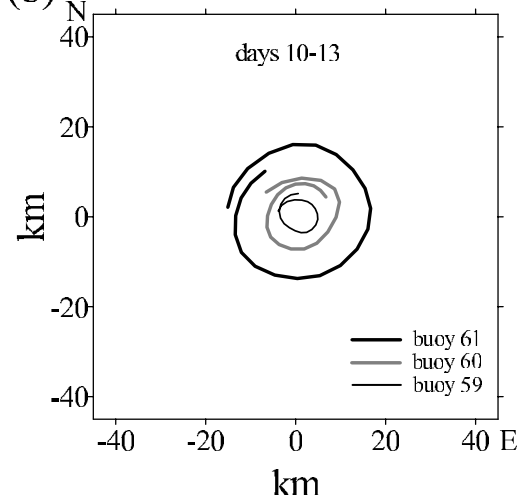

(c)

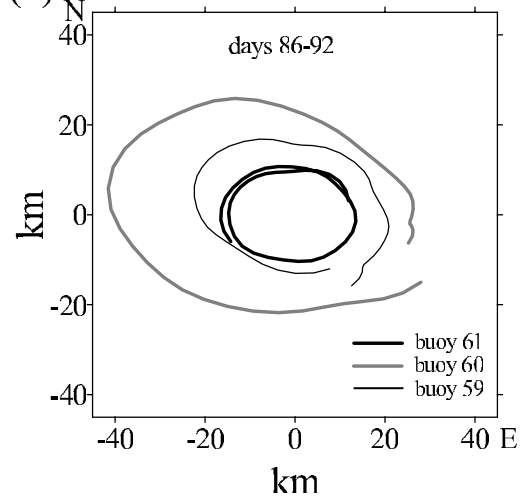

Figure 8. (a) Mean orbital radius as a function of time for all three buoys. Buoys orbits for (b) days 1013 and (c) days 86-92. 
three buoys. It consists on inner near-circular trajectories while the eddy is relatively young, with a typical radius between 5 and $15 \mathrm{~km}$ that depends on the buoy's deployment position, and outer trajectories at later times. The outer trajectories are elliptical, their major axis being zonally oriented and up to about $40 \mathrm{~km}$. Buoy 61 described a total of 56 revolutions during the 199 days time period, with 42 revolutions ( $75 \%$ of the total) during the first 130 days ( $65 \%$ of the time). Buoys 59 and 60 described 32 and 30 revolutions, respectively, during a time period very close to 100 days, in both cases with 16 revolutions (about $50 \%$ of the total) during the first 40 days (about $40 \%$ of the time).

[26] Figure 7 displays the instantaneous and (6 day) mean orbital radius for the three individual buoy trajectories. This mean radius has been obtained by low-pass filtering the instantaneous values ( 3 hour data) with the same 6 day cutoff period we used to calculate the mean trajectory. Because of this smoothing procedure the last 3 days of the time series, when the mean radius appears to decrease, should be ignored. In all cases the instantaneous radius fluctuates around a slowly changing mean value, which indicates that either the buoy trajectory is elliptical or it experiences radial displacements. The buoys sequentially find their place in an outer ring that experiences large radial fluctuations. We may hence grossly talk about an initial buoy regime, with all three buoys located within the inner core where they rotate swiftly and synchronously with small radial fluctuations, and a terminal buoy regime, with all buoys located in the outer ring where they turn slowly while experiencing large radial fluctuations.

[27] The kinematics of the water parcel may be characterized by the buoy's mean radius and radial fluctuations. Buoy 61 , for example, appears to be locked in the early regime till about day 120 , with its mean radius and fluctuations decreasing with time (Figure 7a). This says nothing about the real size of the eddy (which responds to the eddy stage) but simply indicates that the buoy moves in the interior region of the eddy, its mean motion actually converging toward the eddy's center. After approximately day 130, buoy 61 moves away from the eddy center, as evidenced by the rapid increase in the buoy's mean radius (Figure 7a). The SST images (Figures $2 \mathrm{i}$ and $2 \mathrm{j}$ ) clearly show that such increase is the result of the merging of eddies A1 and AT. After this interaction the buoy finds itself rotating with much greater mean radius and radial fluctuations.

[28] Figure 8a presents a comparison of the temporal evolution of the mean orbital radius for all three buoys. Recall that initially buoys 59, 60 and 61 were the inner, intermediate and outer buoys, respectively. During the first 35 days buoy 61 remains as the outer buoy but by day 20 buoys 59 and 60 have exchanged positions. Between days 40 and 50 buoy 60 increases its revolution radius and occupies the outermost position, and buoy 59 also moves out and reaches the same radial position as buoy 61 . Near day 85 buoy 59 moves further out and reaches buoy 60 . Meanwhile buoy 61 , now the innermost buoy, describes orbits progressively smaller till about day 120 , when it begins an outward displacement such that by day 150 its orbital radius has tripled. We also show the buoys orbits between days 10 and 13 (Figure 8b) and between days 86 and 92 (Figure 8c).
The buoys' initial behavior is characteristic of solid body type rotation, with the three buoys retaining their deployment radial order. By day 86 all buoys have exchanged positions and the two outermost buoys (60 and 59) rotate much slower than the inner buoy (61).

[29] From the orbital radius and the tangential velocity we may indirectly estimate the angular velocity $\omega$. Figure 9 presents, for all buoys, the instantaneous and mean rotation periods (inversely proportional to angular velocity). These periods should be coherent with the results from the wavelet analysis (Figure 5): buoy 61 (Figure 9a), for example, shows that from days 10 to 35 the period is 2.5 days $(\omega=$ $2.9 \times 10^{-5} \mathrm{~s}^{-1}$ ) and between days 50 and 120 it is 3.5 days $\left(\omega=2.1 \times 10^{-5} \mathrm{~s}^{-1}\right)$. A comparison of Figures 7 and 9 also serves to appreciate several features. First, the reduction in the mean orbital period of buoy 60 between days 20 and 35 takes place at a time when the mean radius of this buoy is only about $7 \mathrm{~km}$, indicating that the center of the inner core initially rotates at an even faster pace (Figures $7 \mathrm{~b}$ and $9 \mathrm{~b}$ ). Second, the behavior of the buoys in the outer ring, buoy 60 after approximately day 40 (Figures $7 \mathrm{~b}$ and $9 \mathrm{~b}$ ) and buoy 59 after approximately day 85 (Figures 7c and 9c), appears uncoupled to the motion of the buoys that remain within the inner core. Finally, the mean orbital radius of buoy 61 shortens until day 120 (Figure 7a) but between approximately days 35 and 120 this is accompanied by an increase of the orbital mean period, implying that the inner core slowly spins down during the eddy mature stage.

\section{Discussion}

\subsection{Generation Frequency}

[30] The images available for the July-November 1998 period evidence the generation of three anticyclonic and one cyclonic eddies. Anticyclonic eddy A1 (tracked by the buoys) appears on the 9 July image (Figure 2a) as an already well developed eddy, which suggests that it was generated about 2 or 3 weeks earlier [Basterretxea et al., 2002], and remains visible during the whole sequence of SST images. The second anticyclonic eddy A2 is first clearly appreciated in the 16 August image (Figure 2e), although it appears faintly in the 13 August image (not shown). The third anticyclonic eddy AT shows up as a well develop vortex in the 26 October image (Figure 2i). Cyclonic eddy $\mathrm{C} 1$ is viewed as a new born eddy in the 9 July image (Figure 2a) but disappears by 10 September possibly because of surface veiling through atmospheric heating (Figure 2g). On 29 September (day 91), shortly after all buoys (specially buoys 60 and 59) display important changes in behavior, a cyclonic ripple is seen scratching the northern edge of eddy A1.

[31] Figure 3c shows the main changes in mean speed experienced by buoy 61 . During the generation of eddies A1 and C1 the buoy swiftly drifts south with a mean speed of $4.5 \mathrm{~km} \mathrm{~d}^{-1}$. Afterward the buoy temporally modifies its speed and direction, to the east at $2.7 \mathrm{~km} \mathrm{~d}^{-1}$, possibly because of the repulsion between vortices A1 and C1. Next the buoy moves west with a mean speed of $4.1 \mathrm{~km} \mathrm{~d}^{-1}$ and vortices A2 (when the buoy moves southwest) and AT (when it moves northwest) are generated. We assume that the vortex translates stationary relative to the mean flow 
(a)

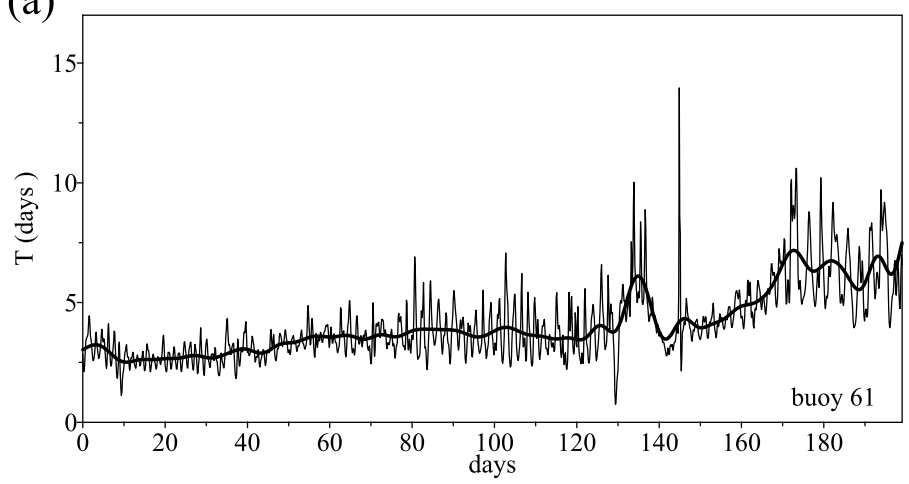

(b)

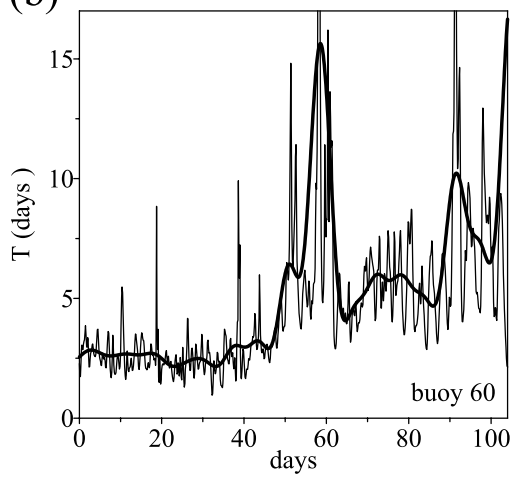

(c)

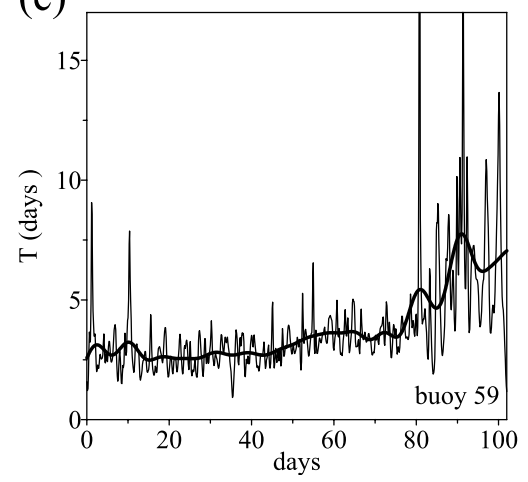

Figure 9. Instantaneous (thin line) and mean (bold line) orbital periods as obtained from the tangential velocity and radial position of the buoys. (a) Buoy 61. (b) Boy 60. (c) Buoy 59.

and use the mean buoy speed (at the times when vortices were generated, i.e., $4.3 \mathrm{~km} \mathrm{~d}^{-1}=0.05 \mathrm{~m} \mathrm{~s}^{-1}$ ) as an estimate for the speed of the southward mean flow impinging onto the islands $U$ [Pingree, 1996; Flament et al., 1996; Kennan and Flament, 2000]. We may hence calculate the Strouhal number $S$, defined as [e.g., Piccirillo and Vanatta, 1993]

$$
S=\frac{f D}{U},
$$

where $f$ is the frequency of generation of the vortices and $D$ is the diameter of the obstacle (in the case of Gran Canaria this being, to a good approximation, a cylindrical obstacle). From a shedding period of 50 days (roughly the time differences between the generation of $\mathrm{A} 1$ and $\mathrm{A} 2$, or between A2 and AT) we may estimate $f=1 / T=2.3 \times$ $10^{-7} \mathrm{~s}^{-1}$. Using this value, $U=0.05 \mathrm{~m} \mathrm{~s}^{-1}$, and $D=$ $50 \mathrm{~km}$ gives $S=0.23$. If we consider uncertainties in the shedding period and mean speed of \pm 15 days and $\pm 0.01 \mathrm{~m} \mathrm{~s}^{-1}$, respectively, then the Strouhal number ranges between 0.15 and 0.41 . These values are in reasonable good agreement with the experimental value $S=0.21$ that holds, over a large range of Reynolds values, for vortices shed by cylindrical obstacles [Countanceau and Bouard, 1977; Gerrard, 1978; Piccirillo and Vanatta, 1993]. This simple calculation supports the hypothesis that these geophysical vortices are topographically generated, i.e., through differential bottom stress with the island's sloping sides.
[32] We may wonder what is the Reynolds number Re during the generation of these vortices. This number is defined as

$$
R e=\frac{U D}{\nu},
$$

where $v$ is the horizontal eddy viscosity coefficient, the main uncertainty in this calculation. Values reported for $v$ are quite diverse, as large as $250 \mathrm{~m}^{2} \mathrm{~s}^{-1}$ in an intense western boundary current [Bower et al., 1985] and $22 \mathrm{~m}^{2} \mathrm{~s}^{-1}$ within an anticyclonic eddy in the Northeast Atlantic Ocean [Martin et al., 2001], to as low as $3 \mathrm{~m}^{2} \mathrm{~s}^{-1}$ in the eastern North Atlantic subtropical gyre [Ledwell et al., 1993] and $0.6 \mathrm{~m}^{2} \mathrm{~s}^{-1}$ at the edge of an anticyclonic vortex in the equatorial Pacific [Lumpkin et al., 2000]. Because of this uncertainty we follow an opposite approach, i.e., we use the lowest Reynolds number necessary for shedding vortices, approximately $R e=60$ [Berger and White, 1972; Countanceau and Bouard, 1977; Gerrard, 1978], and estimate the maximum viscosity coefficient to approximately be $v=40 \mathrm{~m}^{2} \mathrm{~s}^{-1}$. This value is of the same order as the maximum diffusion coefficient that may be accounted by our observations of radial convergence $\left(25 \mathrm{~m}^{2} \mathrm{~s}^{-1}\right.$, see below).

\subsection{Stability}

[33] A remarkable feature of the anticyclonic eddy is that its initial angular velocity, $\omega=-3 \times 10^{-5} \mathrm{~s}^{-1}$, marginally satisfies the condition $\omega>-f / 2=3.3 \times 10^{-5} \mathrm{~s}^{-1}$, where $f$ is 
the planetary vorticity. Buoy 60 actually shows a slight increase of this angular velocity, to almost exactly the condition $\omega=-f / 2$, while it approaches the eddy center between days 20 and 35 (Figure 9b). A fraction of this change in relative vorticity may be attributed to the buoy's initial southward motion, i.e., as the water parcel moves south $1^{\circ}$ of latitude the condition of constant total vorticity would imply a decrease in the revolution period of about 0.2 days, but the observed change is almost 0.5 days.

[34] The above observation is consistent with the stability condition for vortices [e.g., Gent and McWilliams, 1986; Hopfinger and van Heijst, 1993]:

$$
\left(f+2 \omega+r \frac{\partial \omega}{\partial r}\right)(f+2 \omega) \geq 0 .
$$

During its early life the anticyclonic eddy experiences nearsolid-body-type rotation, i.e., $\partial \omega / \partial r$ is zero within the eddy and positive at the eddy edge. In this case the condition $(f+$ $2 \omega) \geq 0$ imposes the largest possible absolute value of the angular velocity, $|\omega|=f / 2$. This causes that during the generation of an anticyclonic eddy the absolute angular velocity cannot exceed half the planetary vorticity value, otherwise the inertial stability condition is not satisfied and the excess in absolute vorticity is rapidly diffused out (through unstable radial motions). Once the (stable) vortex has been generated, however, there appears to be no mechanism capable of producing the inertially unstable situation, which contributes to its enduring character. We may further pursue this argument to hypothesize that the stability of cyclonic eddies will be controlled by the negative vorticity gradient, $\partial \omega / \partial r$, at its outer edge. Initial solid-body-type rotation causes very large negative vorticity at the vortex edge that may lead to an unstable situation. Instability would then enhance radial diffusion of vorticity until a stable situation is reached, possibly resulting in a relatively short life span.

[35] The different behavior of cyclonic and anticyclonic vortices in a rotating system has been well recognized by several authors, with the results being very sensible to the model used to represent the radial distribution of angular velocity [Kloosterziel and van Heijst, 1991; Hopfinger and van Heijst, 1993; Matsuura, 1995; Carnevale et al., 1997]. Flierl [1988] considered the case of a barotropic vortex with inner constant angular velocity, surrounded by a constant angular velocity ring with smaller absolute value. He showed that the possibility of centrifugal instability increases as the thickness of the external ring decreases, independently on the sign of the vortex. This coincides with the above ideas for cyclonic vortices, our initial condition being an outer ring of zero thickness, but clearly disagrees for the anticyclonic one where the cyclonic relative vorticity in the outer ring should be an stabilizing factor.

[36] Flierl [1988] has also shown that baroclinic instabilities in vortices may appear only when its horizontal size $L$ is large as compared with the baroclinic deformation radius, $R_{d}$, the condition being $L>4.2 R_{d}$. The deformation radius is a function of the baroclinic mode under consideration, $R_{d}=c_{n} / f$, where $c_{n} \approx N H / n \pi$ is the wave speed of the nth baroclinic mode in the nonrotating ocean, that depends on the buoyancy frequency $N$ and the ocean depth $H$. Using $N=0.01 \mathrm{~s}^{-1}$ as a typical value in the thermocline layers of the Canary Basin (maximum value in the upper thermocline is as large as $0.03 \mathrm{~s}^{-1}$ ) and $H=2500 \mathrm{~m}$ as the mean water depth in the ocean plateau around the Canary Islands, we estimate the speed of the fastest baroclinic mode $(n=1)$ as $c \cong 8 \mathrm{~m} \mathrm{~s}^{-1}$, from which we calculate the longest baroclinic radius to be about $110 \mathrm{~km}$. According to the above criterion this large value assures that the vortex will not develop baroclinic instabilities, something that probably contributes to its temporal persistence.

[37] Inward radial advection may also be an important factor regulating the temporal coherence of the temperature and vorticity fields. Our data shows that during the first 2 or 3 weeks the anticyclonic vortex displays little or null radial velocity (Figure 8a). At this time the initial high radial stability may be sufficient to maintain a coherent vortex, with little exchange at its outer edge, but later on the dominant factor to account for outward diffusion must be mean radial convergence. A steady state balance between the advective, $\partial\left(r u_{r} \omega\right) / \partial r$, and diffusive, $\partial(r K \partial \omega / \partial r) / \partial r$, terms leads to a diffusion coefficient $K \approx R U_{r}$, where $R \equiv L / 2$ and $U_{r}$ is a characteristic value for the radial velocity. We estimate $U_{r}=0.001 \mathrm{~m} \mathrm{~s}^{-1}$ from the mean position of buoy 61 , which approached the eddy center by $10 \mathrm{~km}$ between days 20 and 120 (Figure 7a). Using this value and $R=25 \mathrm{~km}$ shows that radial convergence could handle a steady state with an eddy diffusion coefficient as large as $25 \mathrm{~m}^{2} \mathrm{~s}^{-1}$. Later on radial convergence is weak, or zero, and radial diffusion is the final mechanism responsible for smoothing out the gradients. Notice that such a balance would not be possible for cyclonic vortices because of the additive effects of both outward diffusion and radial divergence. If we use the same diffusion coefficient and (outward) radial velocity values we would find that a cyclonic vortex could last no longer than a few weeks.

\subsection{Vortex Merging}

[38] The generation of mesoscalar vortices, by processes such as baroclinic and barotropic instability of interior or coastal currents, is a recurrent process in the ocean. Since these vortices last relatively long their encounter is more a rule than an exception, as evidenced by field [Cresswell, 1982; Tokos et al., 1994; Flament et al., 2001; Pingree and Sinha, 2001], laboratory [Nof and Simon, 1987; Griffiths and Hopfinger, 1987; Cerretelli and Williamson, 2003], and numerical [Verron and Valcke, 1994; Valcke and Verron, 1997; von Hardenberg et al., 2000] observations on the merging of like sign vortices. The physical basis for this interaction is the encounter of water parcels with equal potential vorticity such that their exchange is possible. The kinematic process has an initial phase of fluid exchange as the vortices peel each other, which lasts a few orbital periods, and a second phase of reorganization around a single axis of revolution.

[39] Our observations indeed show that the anticyclone A1 encounters both cyclonic and anticyclonic vortices. As anticipated, every time A1 encounters another anticyclonic vortex a fusion takes place: A1 merges with A2 around day 50 and with AT around day 135. In both cases there are major changes in the buoys' mean speed and trajectory, the buoys' orbital motions, and the position and size of the warm core. During the interaction the whole structure formed by the two anticyclones rotates clockwise such that 
(a)

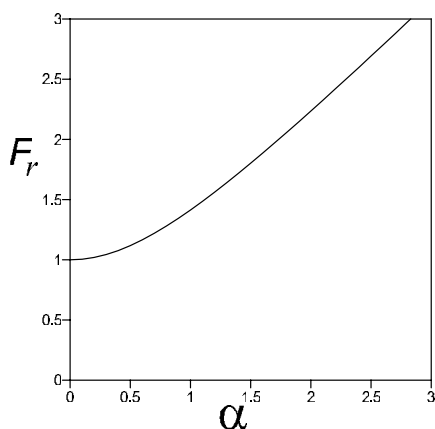

(b)

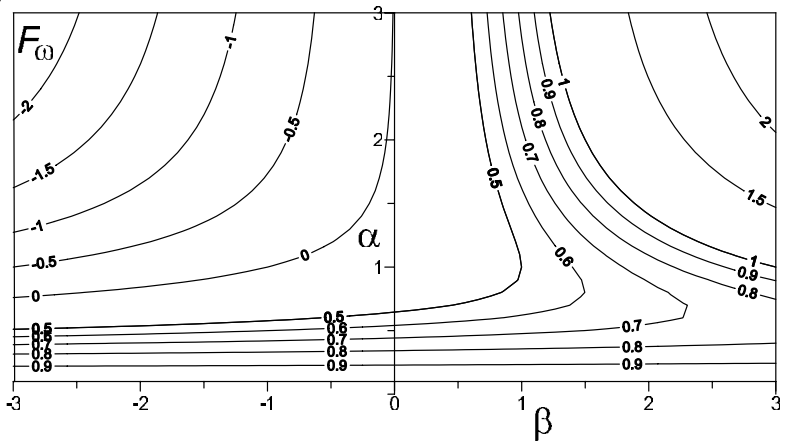

(c)

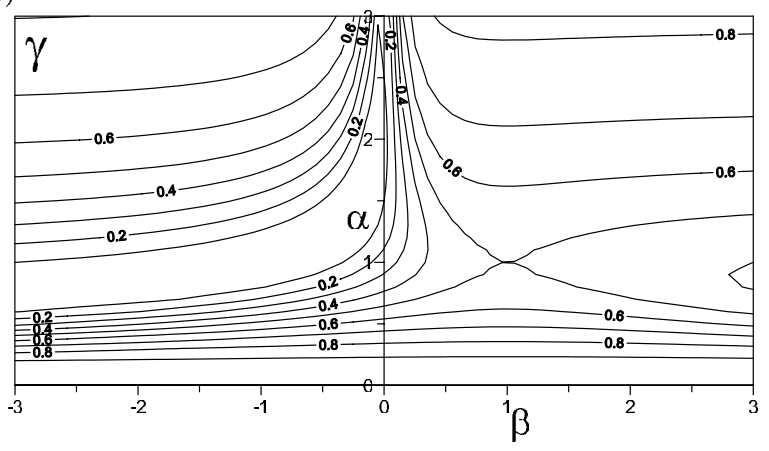

Figure 10. (a) Ratio of the radius of the merged vortex $c$ and the initial vortex $a, F_{r}=r_{c} / r_{a}$, as a function of the ratio of the two merging vortices $b$ and $a, \alpha=r_{b} / r_{a}$. (b) Ratio of the angular velocity of the merged vortex $c$ and the initial vortex $a, F_{\omega}=\omega_{c} / \omega_{a}$, as a function of the ratios of the two merging vortices $b$ and $a, \alpha=r_{b} / r_{a}$ and $\beta=\omega_{b} / \omega_{a}$. (c) Ratio of the mechanic energy of the fused vortex and the mechanic energy of the two merging vortices, $\gamma=E_{c} / E$.

A1 moves northwest, in accordance with laboratory [e.g., Hopfinger and van Heijst, 1993] and numerical [e.g., Cerretelli and Williamson, 2003] experiments.

[40] We also note that the first time A1 encounters cyclonic vortex $\mathrm{C} 1$, at about day 35 , there appears to be some repulsion. This is evidenced by a change in the buoys' mean trajectory and orbital motions, as well as by the position of the warm-core and cold-core features in the SST images, which drift away while the whole structure rotates anticlockwise. However, it does appear that A1 merged with $\mathrm{C} 1$ when they met again at a later time, at about day 85 . The interaction actually took place between the rather large and mature anticyclonic vortex A1, which had previously interacted with other vortices, and the much smaller cyclonic vortex $\mathrm{C} 1$. The key factor in this cyclonic- anticyclonic interaction apparently was the maturity of the anticyclonic vortex, which probably has a positive vorticity outer ring of size comparable to the radius of $\mathrm{C} 1$.

[41] The details of the initial horizontal vorticity distribution is critical for the interaction to begin and is possibly also important for the net amount, and distribution, of vorticity in the fused vortex. The description of these features goes beyond the objectives of this paper but we wonder whether a simple approach, where we allow equal sign and opposite sign vortices to merge, may render consistent results. We idealize the vortices as revolving cylinders, which appears to be a reasonable approximation for deep penetrating topographically generated vortices, and follow the arguments by Lumpkin et al. [2000] to impose that after fusion the system conserves both mass and angular momentum.

[42] The mass within an axisymmetric cylinder-type vortex of radius $r_{a}$ and depth $H$ is given by $2 \pi \rho H \int_{0}^{r_{a}} r d r=$ $\pi \rho H r_{a}^{2}$. The relative angular momentum of an axisymmetric cylinder-type vortex of radius $r_{a}$ and depth $H$ is given by $2 \pi \rho H \int r^{2} v(r) d r=\left(\pi \rho H \omega_{a} r_{a}^{4}\right) / 2$, the equality following for the case of a cylinder rotating as a solid body, $v(r)=\omega_{a} r$. Hence conservation of mass and momentum imply the following:

$$
\begin{gathered}
M \equiv r_{c}^{2}=r_{a}^{2}+r_{b}^{2} \\
L \equiv \omega_{c} r_{c}^{2}=\omega_{a} r_{a}^{2}+\omega_{b} r_{b}^{2},
\end{gathered}
$$

where $\omega$ and $r$ represent the angular velocity and radius of the cylinder, and the subindexes $a, b$ refer to the original vortices, and the subindex $c$ to the vortex resulting after the interaction.

[43] The solution of system (4) may be expressed in terms of the ratios between the properties of the resulting vortex, $c$, and one of the original vortices, say $a$ :

$$
\begin{gathered}
F_{r} \equiv \frac{r_{c}}{r_{a}}=\left(1+\alpha^{2}\right)^{1 / 2} \\
F_{\omega} \equiv \frac{\omega_{c}}{\omega_{a}}=\frac{\left(1+\alpha^{4} \beta\right)}{\left(1+\alpha^{2}\right)^{2}},
\end{gathered}
$$

where $\alpha \equiv r_{b} / r_{a}$ and $\beta \equiv \omega_{b} / \omega_{a}$ are the ratios between the radiuses and vorticities of the two original vortices, respectively. Figure 10 presents the distribution of the ratios $F_{r}$ and $F_{\omega}$ as a function of $\alpha$ and $\beta$; from this figure we may also calculate the ratios $r_{c} / r_{b}$ and $\omega_{c} / \omega_{b}$, between the resulting vortex $c$ and the other original vortex $b$, simply by replacing $\alpha$ and $\beta$ by their inverse numerical values.

[44] When one of the initial vortices is much greater than the other then the size ratio between the merged vortex and the small initial vortex is similar to the size ratio between the large and small initial vortices, i.e., large $\alpha$ implies $F_{r} \cong \alpha$ and small $\alpha$ implies $F_{r} \cong 1$ (Figure 10a). Figure 10b shows that the size of the vortices also controls to a great extent the resulting vorticity ratios, i.e., when $\alpha$ is large the vorticity ratio between the merged vortex and the 
Table 1. Observed and Predicted Values for the Vortex Merging Parameters ${ }^{\mathrm{a}}$

\begin{tabular}{l} 
Interaction $(a+b)$ \\
\hline
\end{tabular}

small initial vortex is similar to the vorticity ratio between the large and small initial vortices, $F_{\omega} \cong \beta$, and when $\alpha$ is small the vorticity ratio between the merged vortex and the small initial vortex is close to one, $F_{\omega} \cong 1$. Only when $\alpha$ is about 1 (roughly between 0.5 and 2 ) then the initial vorticity ratio $\beta$ is what controls the outcome. For example, if both interacting vortices have equal radius and vorticity then the radius of the resulting vortex increases by a factor $\sqrt{2}$ but its vorticity decreases by a factor 0.5 , while if the initially interacting vortices have equal radius and opposite vorticity their merging cancels out. Further note that the case $\beta=1$ (two equal vortices) gives, as expected, the same result whether we use $\alpha$ or $1 / \alpha$.

[45] It is convenient to examine if the above results are energetically feasible, i.e., the total energy of the merged vortex must be equal or less than the total energy of the two original vortices. The total energy of an axisymmetric vortex of radius $r_{a}$ is the result of its potential and kinetic energies. The potential energy is given by $2 \pi \rho \int_{0}^{r_{a}} \int_{0}^{H} g z r d r$ $d z=\left(\begin{array}{lll}\pi \rho & g H^{2} & r_{a}^{2}\end{array}\right) / 2$. The kinetic energy is given by $\pi \rho H \int_{0}^{r_{a}} v^{2}(r) r d r=\left(\pi \rho H \omega_{a}^{2} r_{a}^{4}\right) / 4$, again assuming solid body rotation $v(r)=\omega_{a} r$.

[46] Conservation of potential energy reduces to mass conservation, so conservation of kinetic energy $E$ would require

$$
E \equiv \omega_{c}^{2} r_{c}^{4}=\omega_{a}^{2} r_{a}^{4}+\omega_{b}^{2} r_{b}^{4}
$$

Using the above definitions the initial energy $E$ is given by

$$
E=\left(1+\alpha^{4} \beta^{2}\right) \omega_{a}^{2} r_{a}^{4}
$$

However, if we substitute the definitions for $\alpha$ and $\beta$, and relations (5), into the left-hand side of relation (6), we get that a fused vortex which conserves mass and angular momentum has an energy

$$
E_{c} \equiv \frac{\left(1+\alpha^{4} \beta\right)^{2}}{\left(1+\alpha^{2}\right)^{2}} \omega_{a}^{2} r_{a}^{4}
$$

that is not equal to $E$. Comparing (6b) and (7) the requirement that the kinetic energy of the merged vortex does not increase $\left(E_{c} \leq E\right)$ reduces to

$$
\gamma \equiv \frac{E_{c}}{E}=\frac{\left(1+\alpha^{4} \beta\right)^{2}}{\left(1+\alpha^{4} \beta^{2}\right)\left(1+\alpha^{2}\right)^{2}} \leq 1
$$

[47] Figure 10c shows the distribution of the energy conversion parameter $\gamma$ for the same domain as in Figure 10b. The parameter is everywhere less than one, as it should from the requirement that merging has to take place without external energy input. The value is close to one when $\alpha$ is much smaller or larger than one, i.e., when one of the vortices is much smaller than the other. If the two interacting vortices have equal angular velocity $(\beta=1)$ the minimum value $(\gamma=0.5)$ corresponds to equal-size vortices $(\alpha=1)$, the result being independent on whether we use $\alpha$ or $1 / \alpha$. Maximum energy loss $(\gamma \cong 0)$ corresponds to the merging of two vortices of opposite sign and similar size, as expected from Figure 10b which tells us that such a fusion leads to a vanishing vortex.

[48] We may use the satellite images to obtain an estimate for $\alpha$ every time two vortices interact. For anticyclonic vortices an estimate for $\beta$ is obtained by simply assuming that after generation its angular velocity is $-f / 2$ (to fulfill the inertial stability criterion). For the cyclonic vortex we have no simple way to estimate $\beta$ so we arbitrarily chose $\omega=f / 2$. With these simple criteria we have the values shown in Table 1, the agreement between predictions and observations being encouragingly good. The table also presents the predictions for the energy conversion parameter $\gamma$, the results suggesting that relatively little energy is lost during the observed merging events.

[49] Another issue to consider is the merging time, $T_{m}$, or time that takes after two vortices interact for solid-bodytype rotation to resume. Estimates of this time, as obtained from the wavelet analysis of the buoy's 61 zonal velocities, are about 5-10 days for the A1 + A2 interaction, some 30 days for $\mathrm{A} 1+\mathrm{AT}$, and just about 5 days for $\mathrm{A} 1+\mathrm{C} 1$. The merging time between the anticyclonic vortices increases as A1 revolves more slowly, a plausible result if we expect the merging time to be of the order of several revolution periods. The merging of the anticyclonic and cyclonic vortices $(\mathrm{A} 1+\mathrm{C} 1)$ is much faster, possibly because the relevant timescale is not the revolution period of A1's inner core but some equivalent revolution period for its outer ring.

\subsection{Long-Term Evolution}

[50] The wavelet analysis indicates that at early times the three buoys, located at different radial positions, display near-solid-body rotation with a dominant period about 2.5 days. At this early stage the vortex is quite circular and radial fluctuations are small, so the peak energy remains sharp, although its intensity may decrease as the buoys converge toward the eddy center. Every time the eddy interacts with another mesoscalar structure the buoys suffer large radial displacements, ending up at a similar or different radial position, or even leaving the eddy. An outward 


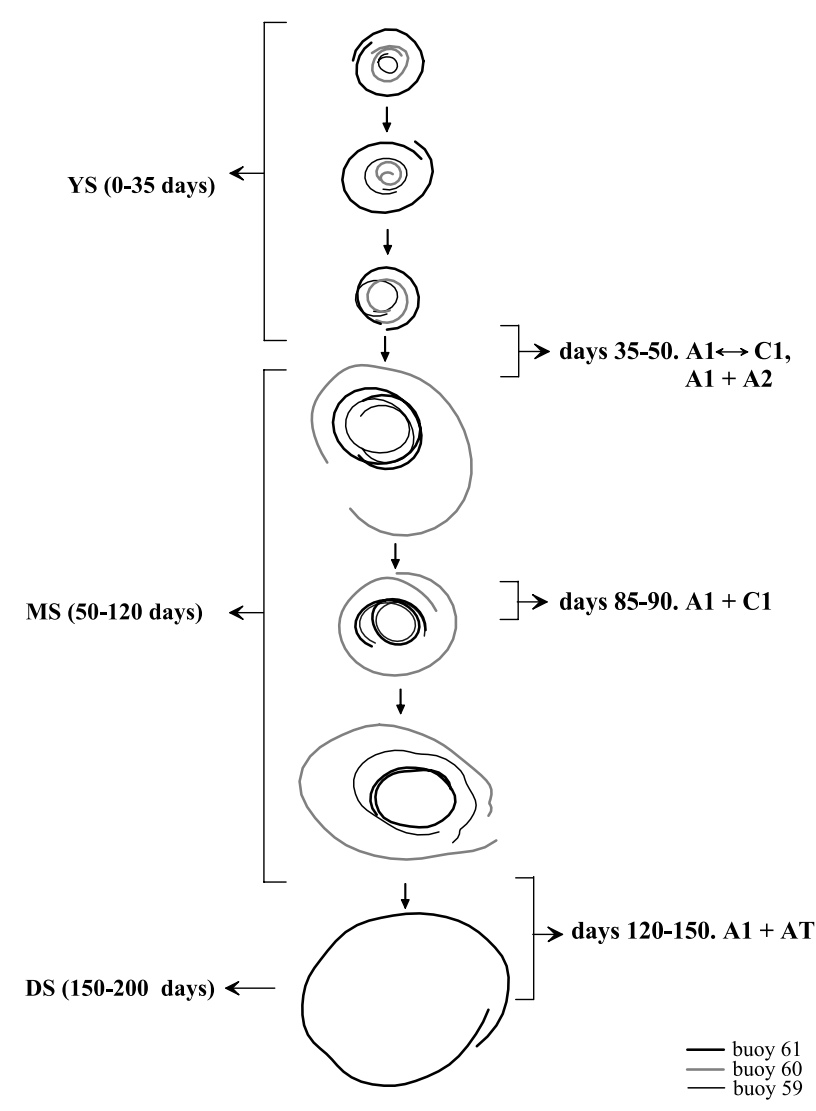

Figure 11. Schematic of the eddy's life history as inferred from the buoys' trajectories. Abbreviations are as follows: YS (young stage), the buoys mean radius and period are approximately constant; MS (mature stage), the buoy found in the eddy core approaches the center as it smoothly slows down, while those at the periphery show a greater decrease in their rotation rate; DS (decay stage), the buoys have escaped from the eddy core, which no longer is well defined, and both the radius and period increase greatly.

displacement causes an increase in the intensity of the peak energy while a sharper peak corresponds to a more stable rotation rate. At these posterior stages, and possibly reinforced by the dissipative processes during the interactions, an outer ring is clearly evident (as in laboratory experiments by Griffiths and Hopfinger [1987] and Cerretelli and Williamson [2003]). We note that all our buoys leave the eddy when they are at a radial position of about $30-35 \mathrm{~km}$, i.e., less than twice the initial eddy radius.

[51] The buoys' orbits complement the wavelet analysis. During the first 40 days all buoys maintain a roughly constant mean orbital radius (between 7 and $15 \mathrm{~km}$ depending on the radial deployment position) and rotation rate (about 2.5 days period). Inward radial motion, as suggested by the trajectory of buoys 60 and 61 , possibly helps vorticity and heat (and the buoys) to stay within the eddy core. Between days 40 and 100 buoy 61 remains in the eddy core while the other two buoys sequentially move to the outer core, where they rotate more slowly. Throughout all this time the mean radius of buoy 61 decreases (some $10 \mathrm{~km}$ in 100 days), indicating an inward radial velocity of $\sim 0.001 \mathrm{~m} \mathrm{~s}^{-1}$. After about 100 days only buoy 61 traces the eddy. This buoy maintains a near constant revolution period approximately between days 50 and 140 , but afterward it experiences a large increase in its rotation period and radius (related to its interaction with vortex AT, Figures $2 \mathrm{i}$ and $2 \mathrm{j}$ ).

[52] In Figure 11 we propose an idealized evolution of the eddy, or eddy life history, in terms of the progressive redistribution of vorticity. Time is taken to be zero when the generation process has already finished and the eddy is fully developed, i.e., approximately day 0 in our data set. During the young stage the eddy core, or central portion of the eddy that rotates as a solid body, practically occupies the whole eddy. After some time the eddy reaches a mature stage with an eddy core that has reduced its size, but retains most of its initial angular velocity, while the eddy's periphery has considerably slowed down. After sufficient elapsed time the eddy reaches a decay stage, yet with a vorticity maximum but its value much smaller than the initial one. The transition from one stage to another, which for an isolated vortex would be a smooth process, appears to speed up as the result of the interaction with other vortices.

[53] Two remarkable characteristics of the eddy long-term evolution are its intermittent pulsation and the existence of radial convergence. The buoys appear to pulsate (their mean orbital radius shrinks and stretches) in two different modes (Figure 7). The first mode consists of relatively rapid pulses of small amplitude probably related to the eddy's ellipticity, such that during one revolution the buoys approach (and separate) its center twice. This agrees with the periodicity of these pulses, initially about 1.5 days and increasing with time, which is roughly half the period of revolution. In the second mode the pulses have much longer period, typically a couple of weeks. One possible explanation for these long pulses is that they may be related to different phases of maximum and minimum eddy eccentricity that develop in vortices [e.g., Cushman-Roisin et al., 1985] and sharp meanders [e.g., Bower, 1989].

[54] As the buoys remain in the inner core they exhibit a mean radial inward velocity of $\sim 0.001 \mathrm{~m} \mathrm{~s}^{-1}$, which implies mean downwelling in agreement with observed patterns of productivity and biomass in the Canary Basin [Arístegui et al., 1997]. To estimate this vertical velocity we consider a cylindrical (axisymmetric) frame of reference and write the continuity equation as follows:

$$
\frac{1}{r} \frac{\partial\left(r u_{r}\right)}{\partial r}+\frac{\partial u_{z}}{\partial z}=0,
$$

where $u_{r}$ and $u_{z}$ are the mean radial and vertical velocity components, respectively. Double integration in the radial, from $r=0$ to $r=R=D / 2$, where $u_{R} \equiv u_{r}(r=R) \cong 0.001 \mathrm{~m} \mathrm{~s}^{-1}$, and vertical coordinates, from $z=0$ to $z=H$, where $u_{z}(z=D) \cong 0$, leads to the following approximate expression:

$$
u_{0}=\frac{2 u_{R} H}{R},
$$

where $u_{0} \equiv u_{z}(z=0)$. Using $H=500 \mathrm{~m}$ and $R=25 \mathrm{~km}$ gives an estimate for the vertical velocity of $3.5 \mathrm{~m} \mathrm{~d}^{-1}$. This estimate is of the same order as observations for epipycnal upwelling within an anticyclone in the North Atlantic [Martin and Richards, 2001] and epipycnal upwelling/ downwelling patches in a quasi permanent eddy in the 
western Mediterranean Sea [Viudez et al., 1996]. Notice that for a steady state solution to exist a cross-vortex circulation cell is necessary, which implies diapycnal velocities of this same order [Rodríguez-Santana et al., 1999, 2001].

\section{Conclusions}

[55] We have used data from three buoys and synchronous SST images to show that an anticyclonic eddy, topographically generated by Gran Canaria island, survived as a coherent structure for at least 7 months and stretched into the interior ocean as far as $500 \mathrm{~km}$ in a region of intense mesoscalar activity. The buoys revolve within the warm eddy that moves southwestward, two of them over 100 days and the third one for almost 200 days. The superposition of the buoys' trajectories onto SST images confirms that the anticyclonic eddy, when isolated from other structures, increases its size slowly with time. The interaction of the eddy with other structures, however, usually leads to merging and a major change in the structure and behavior of the fused eddy. The warm SST signal remains identifiable during practically all 7 month anticyclone's track, its radius never exceeding about $40 \mathrm{~km}$, roughly the terminal orbital radius of the buoy that keeps tracking the vortex during almost 200 days.

[56] From the SST images we appreciate that during 4 months a total of one cyclonic and three anticyclonic eddies were generated. These eddies were generated at times when the mean current speed was over $4 \mathrm{~km} \mathrm{~d}^{-1}$ (about $5 \mathrm{~cm} \mathrm{~s}^{-1}$ ). Using these data we estimate the Strouhal number to be 0.23 , rather close to the experimental value for vortices topographically generated by a cylindrical obstacle over a large range of Reynolds numbers. Assuming a Reynolds number of 60 we estimate the viscosity coefficient to be no greater than $\nu=40 \mathrm{~m}^{2} \mathrm{~s}^{-1}$.

[57] We clearly differentiate between what we observe (the buoys' behavior or regime) and the eddy's stage, our hypothesis being that a good description of the buoys' behavior gives insight into the characteristics of the eddy as it evolves throughout its different life stages. During the first 40 days all buoys are within the inner core (radius less than $20 \mathrm{~km}$ ) where they experience solid body rotation (2.5 days period), with inward mean radial velocity and moderate radial fluctuations. Two buoys move off the eddy's core, at about days 40 and 80 , roughly doubling their revolution radius, halving their revolution rate $(5-$ 6 day period), and displaying large radial fluctuations. The uncoupled behavior of these outer buoys points at the existence of an outer ring that rotates much more slowly than the inner core. After 105 days following deployment only one buoy (61) keeps tracking the eddy. This buoy remains within the eddy's core between days 40 and 135 , slowing down to a 3-4 day period while maintaining the inward radial velocity. By day 135 this last buoy moves off the eddy core and further slows down (period over 6 days) while experiencing large radial fluctuations that last till day 199.

[58] The buoys' trajectories illustrate that the eddy would evolve slowly between different stages, if it were not for the triggering action of the interactions with other mesoscalar structures. A young stage, where most of the eddy core retains its initial rotation rate (period of 2.5 days), lasts $1-$ 2 months. After its interaction with another anticyclonic eddy the periphery of the eddy further slows down, the outer ring is enhanced, and the eddy definitely enters into a mature stage. During this stage the water parcels maintain inward radial convergence and experience significant radial fluctuations. The eddy merges again with an anticyclonic eddy near day 140, this one shed from Tenerife, and enters into a decay stage which is characterized by rather large fluctuations and a slow rotation rate (period over 6 days).

[59] Our observations suggest that, for an isolated eddy, vorticity (and heat) diffusion is small and the region with solid-body-type rotation decreases quite slowly with time. The reason for this behavior may lie on the inertially stable character of relatively weak, $|\omega|<f / 2$, anticyclonic vortices. It may also be reinforced because of the competing effects of radial convergence against outward radial diffusion. A simple calculation shows that the observed inward radial velocity, $0.001 \mathrm{~m} \mathrm{~s}^{-1}$ lasting during 100 days, may account for a diffusion coefficient as large as $\nu=25 \mathrm{~m}^{2} \mathrm{~s}^{-1}$. This value is of the same order as the maximum estimate $\left(40 \mathrm{~m}^{2} \mathrm{~s}^{-1}\right)$ and emphasizes the important role of radial convergence on the eddy's long temporal coherence. On the other hand, since SST images suggest that cyclonic vortices are less persistent it is likely that they are characterized by radial divergence [Aristegui et al., 1997], which would play a diffusing role. Another simple possibility, not to be discarded, is that cyclonic vortices become less visible as a result of surface heating veiling the SST signal.

[60] A major characteristic in the vortex life is that it experiences severe interactions with other mesoscalar structures, both cyclones and anticyclones. During each interaction the buoys experience large radial fluctuations and, in most cases, one of them ends up in the outer ring. Fusion between anticyclonic vortices takes place in two occasions. Fusion between opposite sign vortices only appears to take place in one occasion, between our relatively old anticyclonic vortex and a young cyclonic vortex, the former having an inner core and outer ring with opposite vorticity signs. A simple model where two cylindrical vortices fuse conserving both mass and momentum provides reasonable good agreement with the observations. Application of this model to our observations suggests that relatively little kinetic energy is released during the fusion.

[61] In this paper we have attempted to describe a quite complex structure through a rather limited window. Besides the limitations in the SST and buoy data we must recall that the eddy had a complex baroclinic structure, as a result of the underlying stratification, and was not an isolated feature, instead it interacted with other mesoscalar features in the region and was atmospherically forced throughout its lifetime. Despite so, the data have provided many hints to the mean and fluctuating behavior of water parcels within the eddy. The striking coherence and persistence of these structures, plus its rather frequent generation by oceanic islands and seamounts, make them a very important source of variability and mixing within the ocean. Such longevity is comparable to other surface and subsurface eddies reported for the eastern North Atlantic [Pingree, 1996, 1997]. Further understanding of island-generated vortices requires the planning and development of field experiences 
that assess the evolution of their spatial structure throughout their lifetime, to be accompanied by both process and numerical modeling.

[62] Acknowledgments. We express our gratitude to the officers and crews of R/V Garcia del Cid and A/V Las Palmas and to the technicians of UTM-CSIC for supporting our work at sea. We also want to thank Ana Antoranz, Javier Arístegui, Jesús Cisneros, Egenio Fraile, Bárbara Jiménez, Pilar Pérez, and Joaquín Salvador for their help at different stages of this research and our two anonymous reviewers for their careful and constructive criticism of an original manuscript. This work has been supported by the Spanish government through projects FRENTES (AMB95-0731), COCA (REN2000-1471-C02-02MAR), and BREDDIES (REN20012650/ANT) and the special action MAR1999-1489-E and by the European Union through projects CANIGO (MAS3-CT96-0060) and OASIS (EVK3CT-2002-00073-OASIS). Part of this work was written while J.L.P. was hosted by the Department of Atmospheric and Oceanic Sciences of the University of Wisconsin-Madison, with a grant from the Spanish government (Secretaría de Estado de Educación y Universidades, Ministerio de Educación, Cultura y Deportes).

\section{References}

Arístegui, J., P. Sangrà, S. Hernández-León, M. Cantón, A. HernándezGuerra, and J. L. Kerling (1994), Island-induced eddies in the Canary Islands, Deep Sea Res., Part I, 41, 1509-1525.

Arístegui, J., et al. (1997), The influence of island-generated eddies on chlorophyll distribution: A study of mesoscale variation around Gran Canaria, Deep Sea Res., Part I, 44, 71-96.

Barton, E. D., et al. (1998), The transition zone of the Canary Current upwelling region, Prog. Oceanogr., 41, 455-504.

Barton, E. D., G. Basterretxea, P. Flament, E. G. Mitchelson-Jacob, B. Jones, J. Arístegui, and H. Felix (2000), Lee region of Gran Canaria, J. Geophys. Res., 105, 17,173-17,193.

Basterretxea, G., E. D. Barton, P. Tett, P. Sangrà, E. Navarro-Perez, and J. Arístegui (2002), Eddy and DCM response to wind-shear in the lee of Gran Canaria, Deep Sea Res., Part I, 49, 1087-1101.

Berger, E., and R. White (1972), Periodic flow phenomena, Annu. Rev. Fluid Mech., 4, 313-340.

Borges, R., A. Hernández-Guerra, and L. Nykjaer (2004), Analysis of sea surface temperature time series of the southeastern North Atlantic, Int J. Remote Sens., 25, 869-891.

Bower, A. S. (1989), Potential vorticity balances and horizontal divergence along particle trajectories in Gulf Stream meanders east of Cape Hatteras, J. Phys. Oceanogr., 19, 1669-1681.

Bower, A. S., H. T. Rossby, and J. L. Lilliridge (1985), The Gulf StreamBarrier or blender?, J. Phys. Oceanogr., 15, 24-32.

Boyer, D. L., and M. L. Kmetz (1993), Vortex shedding in rotating flows, Geophys. Astrophys. Fluid Dyn., 26, 43-51.

Carnevale, G. F., M. Briscolini, R. C. Kloosterziel, and G. K. Vallis (1997), Three-dimensionally perturbed vortex tubes in a rotating flow, J. Fluid Mech., 341, 127-163.

Cerretelli, C., and C. H. K. Williamson (2003), The physical mechanism of vortex merging, J. Fluid Mech., 475, 41-77.

Chabert D'Hieres, G., P. A. Davies, and H. Didele (1989), A laboratory study of the lift forces on a moving solid obstacle in a rotating fluid, Dyn Atmos. Oceans, 13, 47-75.

Countanceau, M., and R. Bouard (1977), Experimental determination of the main features of the viscous flow in the wake of a circular cylinder in a uniform translation. Part 1, Steady flow, J. Fluid Mech., 79, 231-256.

Cresswell, G. R. (1982), The coalescence of 2 east Australian Current warm-core eddies, Science, 215, 161-164.

Cushman-Roisin, B., W. H. Heil, and D. Nof (1985), Oscillations and rotations of elliptical warm-core rings, J. Geophys. Res., 90, 11,75611,764 .

Dick, G., and G. Siedler (1985), Barotropic tides in the northeast Atlantic inferred from moored current meter data, Dtsch. Hydrogr. Z., 38, 7-22.

Eugenio, F., J. Marcello, A. Hernández-Guerra, and E. Rovaris (2001), Methodology to obtain accurate sea-surface temperature from locally received NOAA-14 data in the Canary-Azores-Gibraltar area, Sci. Mar., $65,127-137$.

Flament, P., S. C. Kenna, R. Knox, P. Niiler, and R. Bernstein (1996), The three-dimensional structure of an upper ocean vortex in the tropical Pacific, Nature, 382, 610-613

Flament, P., R. Lumpkin, J. Tournadre, and L. Armi (2001), Vortex pairing in an unstable anticyclonic shear flow: Discrete subharmonics of one pendulum day, J. Fluid Mech., 440, 401-409.

Flierl, G. R. (1987), Isolated eddy models in geophysics, Annu. Rev. Fluid Mech., 19, 493-530.
Flierl, G. R. (1988), On the instability of geostrophic vortices, J. Fluid Mech., 197, 349-388.

Gasser, M., J. Salvador, P. Sangrà, and J. L. Pelegrí (2001), Field validation of a semi-spherical Lagrangian drifter, Sci. Mar., 65, 139-143.

Gent, P. R., and J. C. McWilliams (1986), The instability of barotropic circular vortices, J. Fluid Mech., 97, 349-388.

Gerrard, J. H. (1978), Wake of cylindrical bluff bodies at low Reynolds numbers, Philos. Trans. R. Soc. London, Ser. A, 288, 351-382.

Griffiths, R. W., and E. J. Hopfinger (1987), Coalescing of geostrophic vortices, J. Fluid Mech., 178, 73-97.

Grossmann, A., and J. Morlet (1984), Decomposition of Hardy functions into square integrable wavelets of constant shape, SIAM J. Math. Anal., $15,723-736$.

Grossmann, A., J. Morlet, and T. Paul (1985), Transforms associated to square integrable group representations. I. General results, J. Math. Phys., $27,2473-2479$

Hernández-Guerra, A., J. Arístegui, M. Cantón, and L. Nykjaer (1993), Phytoplankton pigments patterns in the Canary Islands as determined using Coastal Zone Colour Scanner data, Int. J. Remote Sens., 14, $1431-1437$

Hernández-Guerra, A., F. López-Laatzen, F. Machín, D. de Armas, and J. L. Pelegrí (2001), Water masses, circulation and transport in the eastern boundary current of the North Atlantic subtropical gyre, Sci. Mar., 65, $177-186$.

Hernández-Guerra, A., et al. (2002), Temporal variability of mass transport in the Canary Current, Deep Sea Res., Part II, 49, 3415-3426.

Hogg, N. G., and H. M. Stommel (1985), The heton, an elementary interaction between discrete baroclinic geostrophic vortices and its implications concerning heat flow, Proc. R. Soc. London, Ser. A, $397,1-20$.

Hopfinger, E. J., and G. J. F. van Heijst (1993), Vortices in rotating fluids, Annu. Rev. Fluid Mech., 25, 241-289.

Kennan, S. C., and P. J. Flament (2000), Observations of a tropical instability vortex, J. Phys. Oceanogr., 30, 2277-2301.

Kloosterziel, R. C., and G. J. F. van Heijst (1991), An experimental study of unstable barotropic vortices in a rotating fluid, J. Fluid Mech., 223, $1-24$.

Ledwell, J. R., A. J. Watson, and C. S. Law (1993), Evidence for slow mixing across the pycnocline from an open-ocean tracer-release experiment, Nature, 364, 701-703.

Lumpkin, R., P. Flament, R. Kloosterziel, and L. Armi (2000), Vortex merging in a $11 / 2$-layer fluid on an f-plane, J. Phys. Oceanogr., 30, $233-242$.

Martin, A. P., and K. J. Richards (2001), Mechanisms for vertical nutrient transport within a North Atlantic mesoscale eddy, Deep Sea Res., Part II, $48,757-773$.

Martin, A. P., K. J. Richards, C. S. Law, and M. I. Liddicoat (2001), Horizontal dispersion within an anticyclonic mesoscale eddy, Deep Sea Res., Part II, 48, 739-755.

Matsuura, T. (1995), The evolution of frontal-geostrophic vortices in a two layer ocean, J. Phys. Oceanogr., 25, 2298-2318.

Morlet, J., G. Arens, I. Fourgeau, and D. Giard (1982), Wave propagation and sampling theory, part I: Complex signal and scattering in multilayered media, Geophysics, 47, 203-221.

Nof, D. (1981), On the beta-induced movement of isolated baroclinic eddies, J. Phys. Oceanogr., 11, 1662-1672.

Nof, D., and L. Simon (1987), Laboratory experiments on the merging of anticyclonic eddies, J. Phys. Oceanogr., 17, 343-357.

Percival, D. B., and A. T. Walden (2000), Wavelet Methods for Time Series Analysis, Cambridge Univ. Press, New York.

Pérez, F. F., L. Mintrop, O. Llinás, M. González-Dávila, C. G. Castro, M. Alvarez, A. Körtzinger, M. Santana-Casiano, M. J. Rueda, and A. F. Ríos (2001), Mixing analysis of nutrients, oxygen and inorganic carbon in the Canary Islands region, J. Mar. Syst., 28, 183-201.

Piccirillo, P. S., and C. W. Vanatta (1993), An experimental study of vortex shedding behind linearly tapered cylinders at low Reynolds numbers, J. Fluid Mech., 246, 163-195.

Pingree, R. D. (1996), A shallow subtropical subducting westward propagating eddy (Swesty), Philos. Trans. R. Soc. London, Ser. A, 354, 102979.

Pingree, R. D. (1997), The Eastern Subtropical Gyre (North Atlantic): Flow rings recirculations structure and subduction, J. Mar. Biol. Ass. U. K., 77, $573-624$

Pingree, R. D., and B. Sinha (2001), Westward moving waves or eddies (storms) on the subtropical/Azores Front near $32.5^{\circ} \mathrm{N}$ ?: Interpretation of the Eulerian currents and temperature records at moorings $155\left(35.5^{\circ} \mathrm{W}\right)$ and $156\left(34.4^{\circ} \mathrm{W}\right)$, J. Mar. Syst., 29, 239-276.

Press, W. H., B. P. Flannery, S. A. Teukolsky, and W. T. Vetterling (1986), Numerical Recipes in Fortran 77: The Art of Scientific Computing, 2nd ed., Cambridge Univ. Press, New York. 
Rodríguez-Santana, A., J. L. Pelegrí, P. Sangrà, and A. Marrero-Díaz (1999), Diapycnal mixing in Gulf Stream meanders, J. Geophys. Res., 104, 25,891-25,912.

Rodríguez-Santana, A., J. L. Pelegrí, P. Sangrà, and A. Marrero-Díaz (2001), On the relevance of diapycnal mixing for the stability of frontal meanders, Sci. Mar., 65, 259-267.

Sangrà, P. (1995), Perturbación de un flujo geofísico por un obstáculo: Aplicación a la isla de Gran Canaria, Ph.D. thesis, 221 pp., Univ. de las Palmas de Gran Canaria, Spain.

Siedler, G., and U. Paul (1991), Barotropic and baroclinic tidal currents in the eastern basins of the North Atlantic, J. Geophys. Res., 96, 22,25922,271 .

Tokos, K. L. S., H. H. Hinrichsen, and W. Zenk (1994), Merging and migration of 2 meddies, J. Phys. Oceanogr., 24, 2129-2141.

Valcke, S., and J. Verron (1997), Interactions of baroclinic isolated vortices: The dominant effect of shielding, J. Phys. Oceanogr., 27, 525-541.

van Camp, L., L. Nykjaer, E. Mittelstaed, and P. Schlittenhard (1991), Upwelling and boundary circulation off northwest Africa as depicted by infrared and visible satellite observations, Prog. Oceanogr., 26, 357-402.

Verron, J., and S. Valcke (1994), Scale-dependent merging of baroclinic vortices, J. Fluid Mech., 264, 81-106.

Viudez, A., R. L. Haney, and J. Tintoré (1996), Circulation in the Alboran Sea as determined by quasi-synoptic hydrographic observations. 2. Me- soscale ageostrophic motion diagnosed through density dynamical assimilation, J. Phys. Oceanogr., 26, 706-724.

von Hardenberg, J., J. C. McWilliams, A. Provenzale, A. Shchepetkin, and J. B. Weiss (2000), Vortex merging in quasi-geostrophic flows, J. Fluid Mech., 412, 331-353.

I. Arregui, Fundación AZTI, Herrera Kaia, Portu aldea z/g, E-20110 Pasaia (Gipuzkoa), Spain. (iarregui@pas.azti.es)

A. Hernández-Guerra, A. Marrero-Díaz, J. M. Martín, A. Martínez, A. Rodríguez-Santana, and P. Sangrà, Departamento de Física, Facultad de Ciencias del Mar, Universidad de Las Palmas de Gran Canaria, E-35017 Las Palmas de Gran Canaria, Spain. (ahernandez@dfis.ulpgc.es; amarrero@dfis. ulpgc.es; jmartin@dfis.ulpgc.es; amartinez@dfis.ulpgc.es; arodriguez@ dfis.ulpgc.es; psangra@dfis.ulpgc.es)

J. L. Pelegrí, Grup d'Oceanografía Física, Institut de Ciències del Mar, Centre Mediterrani d'Investigacions Marines i Ambientals, Consejo Superior de Investigaciones Científicas, Passeig Maritim de la Barceloneta 37-49, E-08003 Barcelona, Spain. (pelegri@icm.csic.es)

A. W. Ratsimandresy, Ente Público Puertos del Estado, Avda. Partenon, 10, E-28042 Madrid, Spain. (andry@ratsimandresy.org) 